Original Research Paper

\title{
Drawdown and Drawup of Bi-Directional Grid Constrained Stochastic Processes
}

\author{
Aldo Taranto and Shahjahan Khan \\ School of Sciences, University of Southern Queensland, Toowoomba, QLD 4350, Australia
}

\section{Article history}

Received: 14-05-2020

Revised: 04-09-2020

Accepted: 14-09-2020

Corresponding Author:

Aldo Taranto

School of Sciences, University

of Southern Queensland,

Toowoomba, QLD 4350,

Australia

Email: Aldo.Taranto@usq.edu.au Aldo.Taranto@credience.com

\begin{abstract}
The Grid Trading Problem (GTP) of mathematical finance, used in portfolio loss minimization, generalized dynamic hedging and algorithmic trading, is researched by examining the impact of the drawdown and drawup of discrete random walks and of Itô diffusions on the Bi-Directional Grid Constrained (BGC) stochastic process for profit $P_{t}$ and equity $E_{t}$ over time. A comprehensive Discrete Difference Equation (DDE) and a continuous Stochastic Differential Equation (SDE) are derived and proved for the GTP. This allows fund managers and traders the ability to better stress test the impact of volatility to reduce risk and generate positive returns. These theorems are then simulated to complement the theoretical models with charts. Not only does this research extend a rich mathematical problem that can be further researched in its own right, but it also extends the applications into the above areas of finance.
\end{abstract}

Keywords: Grid Trading Problem (GTP), Bi-Directional Grid Constrained (BGC), Random Walks, Itô Diffusions, Probability of Ruin, Maximal Drawdown, Maximal Drawup, Discrete Difference Equation (DDE), Stochastic Differential Equation (SDE)

\section{Introduction}

Grid trading involves the simultaneous going long and going short at the current price rate $R_{t}$ (instantly creating a hedged position(s)) and also at fixed width $g$ multiples above and below $R_{t}$. As the price rate propagates through these grid levels which effectively traverses a binomial lattice model over time, assumed to be a discrete 1Dimensional random walk without any loss in generality, but also studied here as a continuous Brownian motion, then the system will close many profitable trades at the next nearest grid level whilst carrying the losing trades open. These open losing trades will eventually be either closed individually when in profit, closed as a system of losing trades when the system is back in profit, or closed as a system of losing trades if the losses grow too large. If the system is not closed in time, it can suddenly lose all or more than the initial equity $E_{0}$, in which case the system is said to be ruined. This is known as the Grid Trading Problem (GTP), emerging from finance and researched here mathematically as a constrained stochastic process. Note that the winning trades accumulate linearly over time regardless of the trend or lack of trend, whilst the losing trades accumulate via the triangular number series as a trend grows linearly over time, as shown in Fig. 1.

Let $R_{t}=R(t)$ be an Itô diffusion given by:

$$
d R(t)=\mu(X, t) d t+\sigma(X, t) d W(t),
$$

which can be simplified for the discrete parts of this paper to:

$$
R(t)=\mu t+\sigma W(t)
$$

where, $W(t), \forall t \in[0, T]$ is a standard Wiener process, $m$ $\in \mathbb{R}$ is the drift (which effects the direction or trend) and $s \in \mathbb{R}, \forall \sigma \geq 0$ is the diffusion (which effects the volatility) parameter over a standard filtered probability space $(\Omega, \mathscr{F}, \mathbb{R})$. This continuous formulation will be followed as much as possible and for our discrete formulations, set $\mu=0$ and $\sigma=1$.

The drawdown $D_{t}$ is defined as the difference between the maximum rate of $R(s)$ and the current rate $R(t)$ at time $t$ as:

$$
D_{t}=\sup _{s \in[0, t]}(R(s))-R(t),
$$

and has been rigorously researched (Graversen and Shiryaev, 2000) and the references therein. This is shown diagrammatically within our discrete binomial lattice model framework in Fig. 2. 


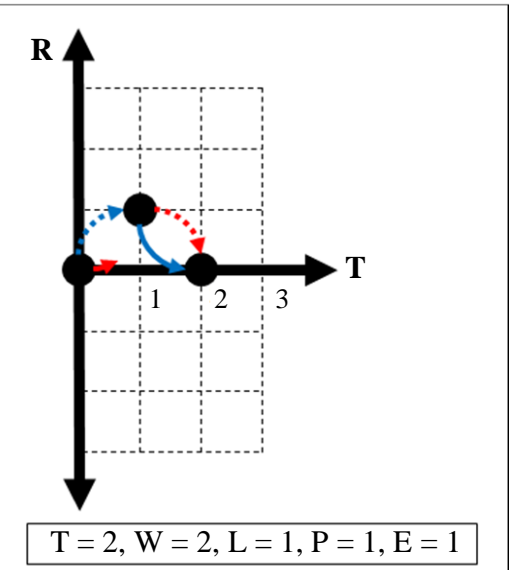

(a)

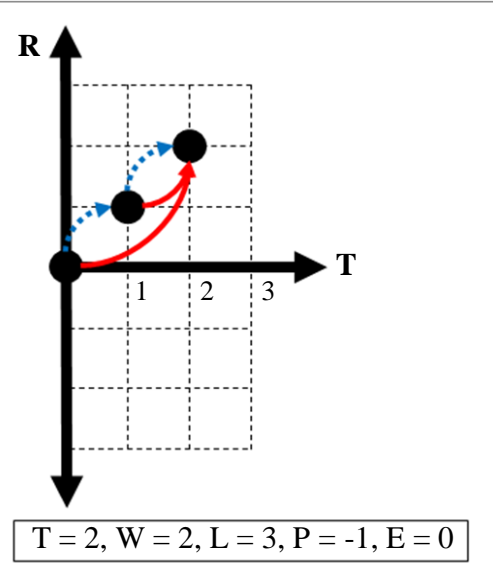

(b)

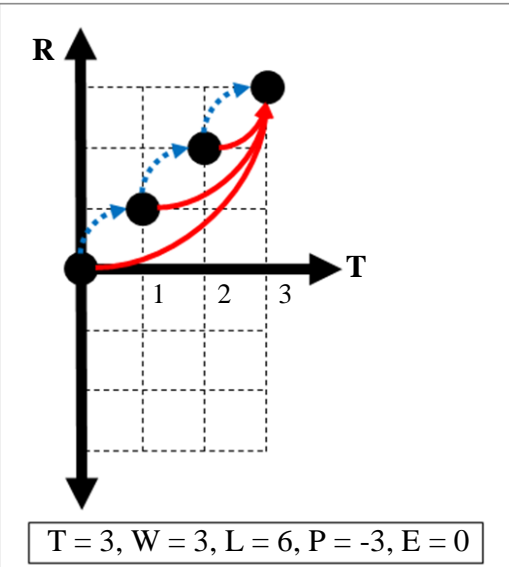

(c)

Fig. 1: GTP and its Profit/Loss Accumulation Process (a). Small Profit (b). Small Loss (c). Larger Loss; $R=$ Rate, $t \in[0, T]=$ Time, $W=$ Winning trades, $L=$ Losing trades, $P=$ Profit, $E=$ Equity, noting that $P \leq 0 \rightarrow E=0$. Dotted lines depict trades closed out in profit at their Take Profit (TP). Solid lines depict open trades in loss that are held until they reach their TP, closed down when the system is back in profit, closed down when loss becomes 'too large' or finally if an account is ruined. Note that the winning trades accumulate linearly over time regardless of the trend or lack of trend, whilst the losing trades accumulate via the triangular number series as a trend grows linearly over time

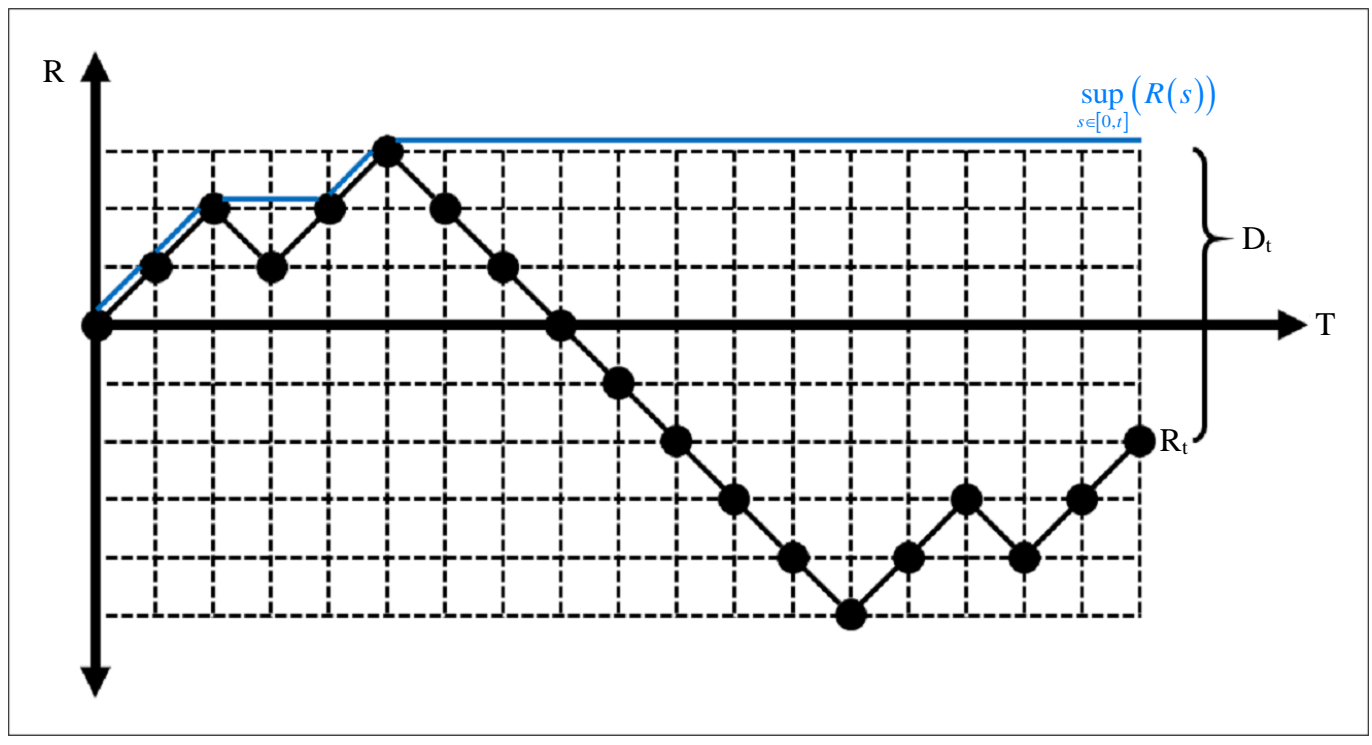

Fig. 2: Drawdown $D_{t}$ of a 1-dimensional discrete random walk as time increases, the previous supremum grows as new maxima are formed

A concept that is often ignored, the drawup $U_{t}$, is defined as the difference between the minimum rate of $R(s)$ and the current rate $R(t)$ at time $t$ as:

$$
U_{t}=R(t)-\inf _{s \in[0, t]}(R(s)),
$$

and is shown in Fig. 3.

The maximal (maximum) drawdown $\bar{D}_{t}$ is commonly used in mathematical finance as an indicator and measure of risk for a stock that follows a particular random process is defined (Magdon-Ismail et al., 2004) as:

$$
\bar{D}_{t}=\sup _{t \in[0, T]}\left(D_{t}\right)=\sup _{t \in[0, T]}\left(\sup _{s \in[0, t]}(R(s))-R(t)\right) .
$$

Note that the corresponding maximal (maximum) drawup $\bar{U}_{t}$ can be expressed as:

$\bar{U}_{t}=\sup _{t \in[0, T]}\left(U_{t}\right)=\sup _{t \in[0, T]}\left(R(t)-\inf _{s \in[0, t]}(R(s))\right)$. 
Aldo Taranto and Shahjahan Khan / Journal of Mathematics and Statistics 2020, Volume 16: 182.197 DOI: $10.3844 / j m s s p .2020 .182 .197$

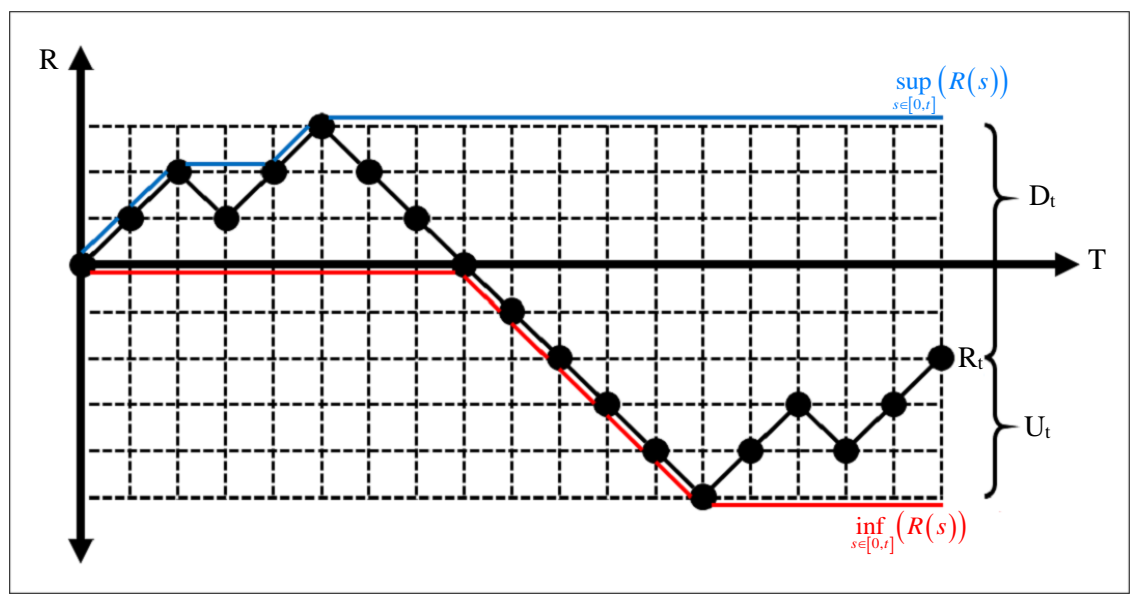

Fig. 3: Drawdown $D_{t}$ and Drawup $U_{t}$ of a 1-dimensional discrete random walk

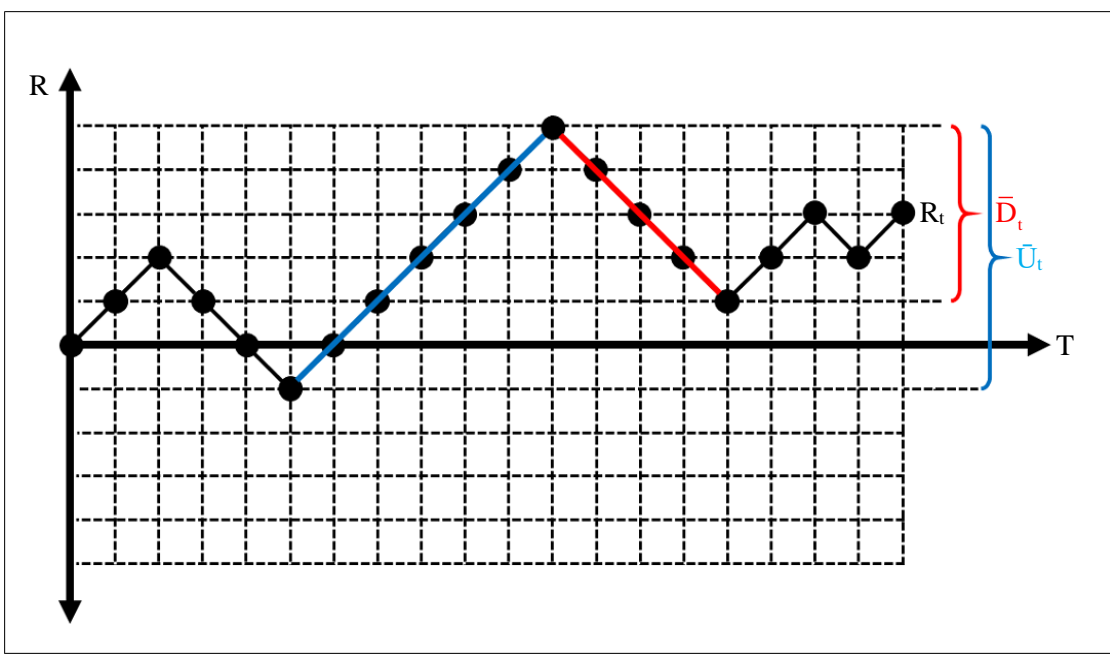

(a)

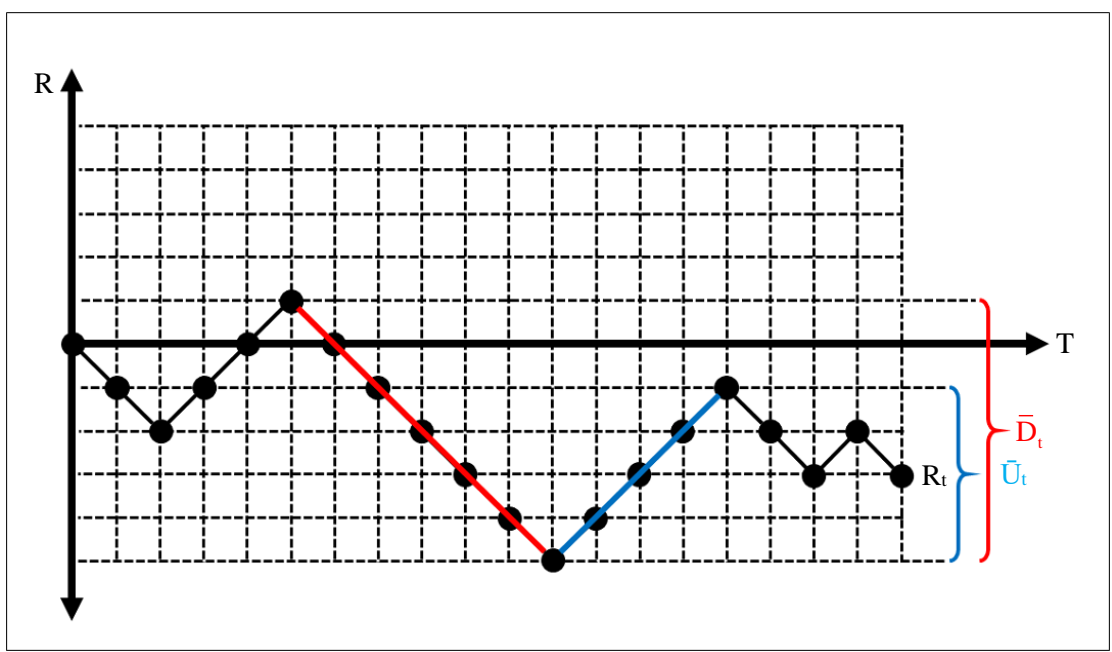

(b)

Fig. 4: Maximal Drawdown $\bar{D}_{t}$ and Maximal Drawup $\bar{U}_{t}$ of two 1-Dimensional Discrete Random Walks (a). Scenario 1: $\bar{D}_{t}<\bar{U}_{t}$; (b). Scenario 2: $\bar{D}_{t}>\bar{U}_{t}$ 
The relationship between the maximal drawup $\bar{U}_{t}$ and the maximal drawdown $\bar{D}_{t}$ is shown in Fig. 4. Informally, the maximal drawdown is the largest drop from a peak to a trough and the maximal drawup is the largest rise from a trough to a peak. It is identified that there is a research gap in the Maximal Drawdown literature as it has only been applied to the trading and investing of 'naked' instruments such as shares, stocks, commodities, which ultimately do not involve derivative instruments. This is where one can only profit from going long, such as watching the shares of IBM go up and down and making a profit when the price $R_{t}$ rises above the initial purchase price $R_{0}$. In contrast to this, the use of more sophisticated trading and investment strategies, in particular the trading of derivative instruments such as Foreign exchange (FX), Contracts For Difference (CFDs), futures, options and many more exotic combinations of these derivatives, means that one can profit from both going long and going short. BiDirectional grid trading involves this second class of instruments and the maximal drawdown and maximal drawup interrelationship is shown in Fig. 4.

From Fig. 4 , it is noted that $\bar{D}_{t}$ does not usually equal $\bar{U}_{t}$ but they can be equal in some scenarios or sample paths. This paper leverages the properties of drawdown, drawup (and the maximum of these to a lesser extent) to develop a Discrete Difference Equation (DDE) theorem and a Stochastic Differential Equation (SDE) theorem of how the GTP evolves over time, along with their corresponding proofs.

\section{Literature Review}

To the best of the authors' knowledge, there is no formal academic definition, other than our own (Taranto and Khan, 2020a; 2020b), of the GTP available within all the references on the subject matter; (Mitchell, 2018; DuPloy, 2008; 2010; Harris, 1998; King, 2010; 2015; AdmiralMarkets, 2017; Work, 2018). Note that these are not rigorous peer reviewed journal papers but instead informal blog posts or software user manuals. Even if there were any academic worthy results found on grid trading, there is a general reluctance for traders to publish any trading innovation that will help other traders and potentially erode their own trading edge.

Despite this, grid trading can be expressed academically as a form of Dynamic Mean-Variance Hedging (Duffie and Richardson, 1991; Černý and Kallsen, 2007). There are many reasons why a firm would undertake a hedge, ranging from minimizing the market risk exposure to one of its client's trades by trading in the opposite direction (Haigh and Holt, 2000), through to minimizing the loss on a wrong trade by correcting the new trade's direction whilst keeping the old trade still open until a more opportune time (Stulz, 2013). In the case of grid trading, it can be considered as a generalized form of hedging of multiple positions simultaneously over time, for the generation of trading profits.

Another academic framework for grid trading is the consideration of the series of open losing trades in a grid system as a portfolio of stocks in the context of MeanVariance Potfolio Optimization problem (Schweizer, 2010; Biagini et al., 2000; Thomson, 2005). This is because a grid trading session involves a basket of winning and losing trades that can be likened to a portfolio of winning and losing shares or stocks. The Merton problem, a question about optimal portfolio selection and consumption in continuous time, is indeed ubiquitous throughout the mathematical finance literature. Since Merton's seminal paper (Merton, 1971), many variants of the original problem have been put forward and have been extensively studied to address various issues arising from economics and finance. For example, (Fleming and Hern'andez-Hern'andez, 2003) considered the case of optimal investment in the presence of stochastic volatility. Davis and Norman (1990; Dumas and Luciano, 1991) and more recently (Czichowsky et al., 2012; Guasoni and Muhle-Karbe, 2013; Muhle-Karbe and Liu, 2012) addressed optimal portfolio selection under transaction costs. Rogers and Stapleton (2002) considered optimal investment under time-lagged trading. Vila and Zariphopoulou (1997) studied optimal consumption and portfolio choice with borrowing constraints.

Turning now to the maximal drawdown of random walks, the research dates back to (Feller, 1951). One of the most comprehensive and mathematically rigorous reviews and advances in the field is due to (Magdon-Ismail et al., 2004) and the references within, where they denote the distribution function for $\bar{D}$ by $G_{\bar{D}}(h)=\mathbb{P}[\bar{D} \geq h]$. It was found that $G_{\bar{D}}(h)$ is given by:

$$
G_{\bar{D}}(h)=2 \sigma^{4} \sum_{n=1}^{\infty} \frac{\theta_{n} \sin \left(\theta_{n}\right)}{\left(\sigma^{4} \theta_{n}^{2}+\mu^{2} h^{2}-\sigma^{2} \mu h\right)} e^{-\frac{\mu h}{\sigma^{2}}}\left(1-e^{-\frac{\sigma^{2} \theta_{n}^{2} T}{2 h^{2}}} e^{-\frac{\mu^{2} T}{2 \sigma^{2}}}\right)+L,
$$

where, $\theta_{n}$ are the positive solutions of the eigenvalue condition, $\tan \left(\theta_{n}\right)=\frac{\sigma^{2}}{\mu h} \theta_{n}$ and $L$ is given by:

$$
L= \begin{cases}0, & \mu<\frac{\sigma^{2}}{h} \\ \frac{3}{c}\left(1-c^{-\frac{\mu^{2} T}{2 \sigma^{2}}}\right), & \mu=\frac{\sigma^{2}}{h}, \\ \frac{2 \sigma^{4} \eta \sinh (\eta) e^{-\frac{\mu h}{\sigma^{2}}}}{\left(\sigma^{4} \eta^{2}-\mu^{2} h^{2}+\sigma^{2} \mu h\right)}\left(1-e^{-\frac{\mu^{2} T}{2 \sigma^{2}} e^{\left.-\frac{\sigma^{2} \eta^{2} T}{2 h^{2}}\right),}}\right. & \mu>\frac{\sigma^{2}}{h}\end{cases}
$$


where, $\eta$ is the unique positive solution of $\tanh (\eta)=$ $\frac{\sigma^{2}}{\mu h} \eta$ and $L$ has three expressions, depending on whether $\mu<\frac{\sigma^{2}}{h}, \mu=\frac{\sigma^{2}}{h}$ or $\mu>\frac{\sigma^{2}}{h}$. This was then extended to random walks on supercritical Galton-Watson trees (Hu et al., 2015). The formulas above are relisted into the following, as this paper is mainly interested in when there is no drift, yielding:

$$
G_{\bar{D}}=\gamma \sqrt{2 x}, L=\frac{3}{c}\left(1-c^{-\frac{\mu^{2} T}{2 \sigma^{2}}}\right) .
$$

Neal (2013) proved the formulas for the averages of the maximum height and the minimum height of a random walk attained before $n$ downward movements occur. Finally, (Hu et al., 2015) also computed the exact value of a negative moment of the maximal drawdown of the standard Brownian motion.

\section{Methodology}

Having introduced the GTP, its profit or loss accumulation process, drawdown, drawup and the maximal of these, together with their associated literature, it is noted that in BGC trading, one needs to know the values of $D_{t}, U_{t}$ at every point in time $t \in T$, as shown in Fig. 5.

Having analysed this is detail, one can now formulate the total losses $L_{t}$ at any time ${ }^{1}$ knowing just $D_{t}$ and $U_{t}$ :

$$
\begin{aligned}
& L_{t}=L_{D_{t}}+L_{U_{t}}=\frac{D_{t}\left(D_{t}+1\right)}{2}+\frac{U_{t}\left(U_{t}+1\right)}{2} \\
& =\frac{1}{2}\left(D_{t}^{2}+D_{t}+U_{t}^{2}+U_{t}\right) .
\end{aligned}
$$

(3.2) has been plotted in Fig. 6 for the first $10 g \mathbb{Z}_{+}$and first $10 \mathrm{~g} \mathbb{Z}_{-}$grid levels, about the discrete $R_{t}$ level.

From Fig. 6, the $R_{t}$ random walk is hence constrained by grid trading to result in the corresponding profit $P_{t}$ and equity $E_{t}$ random walks, where the total loss $L_{t}$ is greatest when the linear combination of $D_{t}$ and $U_{t}$ are the greatest. Having found the dynamics of $L_{t}$, one can now derive the Discrete Difference Equation (DDE) of GTP.

${ }^{1}$ Note that the losses $L_{D_{t}+U_{t}} \neq L_{D_{t}}+L_{U_{t}}$ as:

$$
L_{D_{t}+U_{t}}=\frac{\left[D_{t}+U_{t}\right]\left(\left[D_{t}+U_{t}\right]+1\right)}{2}=L_{D_{t}}+L_{U_{t}}+D_{t} U_{t}
$$

\section{Derivation of BGC DDE of GTP}

\section{Theorem 3.1}

For a Bi-Directional Grid Constrained random walk $R_{t}$ with a value $v$ per grid width, then the change in equity $E$ over time $t$ is given by the following Discrete Difference Equation (DDE):

$$
\begin{aligned}
& E_{t}=E_{0}+v t-v\left(\max _{t \in[0, T]}\left(\sum_{i=0}^{t} \Re_{i}\right)\right)^{2} \\
& +2 v\left(\max _{t \in[0, T]}\left(\sum_{i=0}^{t} \Re_{i}\right)\right)\left(\sum_{i=0}^{t} \Re_{i}\right)-v\left(\sum_{i=0}^{t} \Re_{i}\right)^{2},
\end{aligned}
$$

where, $\mathfrak{R}_{i} \in\{-1,1\}$.

\section{Proof}

In this discrete time framework $t \in \mathbb{Z}_{+} \times \mathbb{Z}$ of our binomial lattice model, one can see that the equity $E_{t}$ at any time $t$ is comprised of the initial equity $E_{0}$, plus the sum of all the winning trades $W_{t}$, minus the sum of all the losing trades $L_{t}$, hence $E_{t}=E_{0}+P_{t}$ where the total profit $P_{t}=W_{t}-L_{t}$. One can now derive the general formula for $E_{t}$, giving:

$$
\begin{array}{lll}
n=0, & E_{0}=E_{0}, \\
n=1, & E_{1}=E_{0}+v-v & \\
\ldots & \cdots & \cdots \\
n=t, & E_{t}=E_{0}+\underbrace{\sum_{i=0}^{t}(v i)}_{W_{t}}-\underbrace{v \frac{D_{t}\left(D_{t}+1\right)}{2}-v \underbrace{}_{t} \frac{U_{t}\left(U_{t}+1\right)}{2}}_{L_{t}}=E_{0}+v \sum_{i=0}^{t} P_{i} \\
=E_{0}+v t-\frac{v}{2}\left(D_{t}^{2}+D_{t}+U_{t}^{2}+U_{t}\right) .
\end{array}
$$

For simplicity, one wishes to substitute $D_{t}$ and $U_{t}$ with a formulation that captures the underlying randomness of $R_{t}$ in terms of the generalized 1Dimensional discrete random walk, where:

$R_{t}=\sum_{i=0}^{t} \Re_{i}$

and:

$D_{t}=v\left(\max _{t \in[0, T]}\left(\sum_{i=0}^{t} \mathfrak{R}_{i}\right)-\sum_{i=0}^{t} \mathfrak{R}_{i}\right)$,
$U_{t}=v\left(\sum_{i=0}^{t} \mathfrak{R}_{i}-\min _{t \in[0, T]}\left(\sum_{i=0}^{t} \mathfrak{R}_{i}\right)\right)$,

where, $\mathfrak{R}_{i} \in\{-1,1\}$ noting that the more general case of $\mathfrak{R}_{i} \in\{-\alpha, \alpha\}$ results in $\alpha \Re$, but is not considered further in this study. Substituting (3.6) into (3.4) gives: 


$$
\begin{aligned}
& E_{t}=E_{0}+v t-\frac{v}{2} D_{t}\left(D_{t}+1\right)-\frac{v}{2} U_{t}\left(U_{t}+1\right) \\
& =E_{0}+t-\frac{v}{2}\left[\left(\max _{t \in[0, T]}\left(\sum_{i=0}^{t} \Re_{i}\right)-\sum_{i=0}^{t} \Re_{i}\right)^{2}\right. \\
& +\left(\max _{t \in[0, T]}\left(\sum_{i=0}^{t} R_{i}\right)-\sum_{i=0}^{t} R_{i}\right) \\
& +\left(\sum_{i=0}^{t} \Re_{i}-\min _{t \in[0, T]}\left(\sum_{i=0}^{t} \Re_{i}\right)\right)^{2} \\
& \left.+\left(\sum_{i=0}^{t} \Re_{i}-\min _{t \in[0, T]}\left(\sum_{i=0}^{t} \Re_{i}\right)\right)\right] .
\end{aligned}
$$

At this stage, two key Lemmas are noted, the proof of which is left to the interested reader to derive:

$$
\begin{aligned}
& \sum_{i=0}^{t}\left(X_{i}\right)+\sum_{i=0}^{t}\left(-X_{i}\right)=0 . \\
& \min _{t \in[0, T]}\left(\sum_{i=0}^{t} X_{i}\right)=-\max _{t \in[0, T]}\left(\sum_{i=0}^{t}\left(-X_{i}\right)\right) .
\end{aligned}
$$

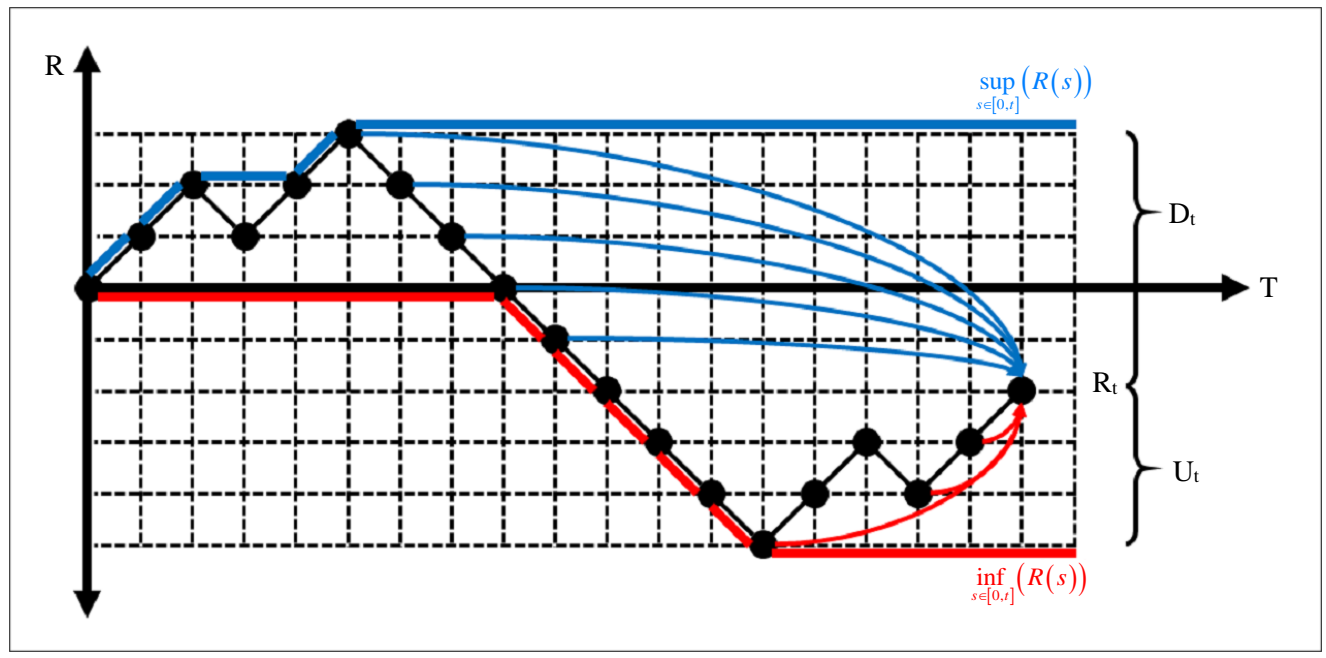

(a)

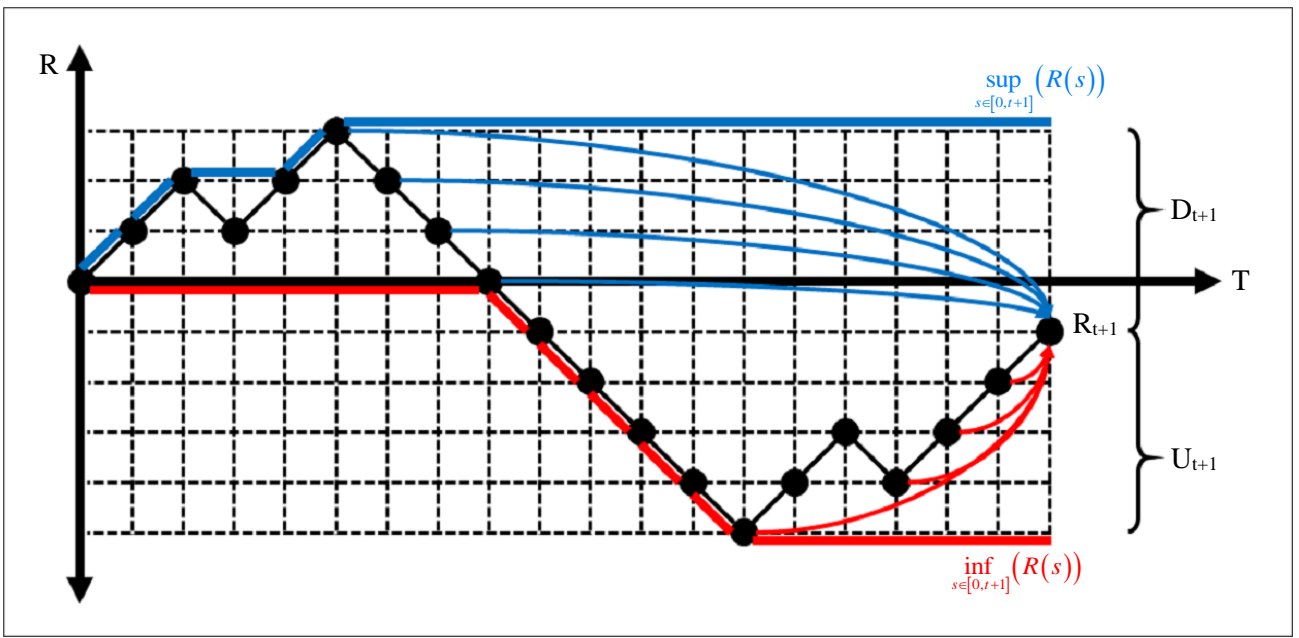

(b)

Fig. 5: Drawdown and Drawup of an Example 1-Dimensional Discrete Random Walk; For simplicity, the winning trades have not been depicted here, which grow linearly over time. It is also noted again that the losses grow via the Triangular number sequence $T_{n}=\frac{n(n+1)}{2} . D_{t}=\sup _{s \in[0, t]}\left((R(s))-R(t), U_{t}=R(t)\right.$ - $\inf _{s \in[0, t]}\left((R(s))\right.$. (a). At $T=t=18, D_{t}=5, U_{t}=3 . E_{0}=0, W_{t}=18, L_{t}=$ $\frac{1}{2}(5[5+1])+\frac{1}{2}(3[3+1])=21, P_{t}=-3, E_{t}=0 ;\left(\right.$ b). At $T=t+1=19, D_{t+1}=4, U_{t+1}=4 . E_{0}=0, W_{t+1}=19, L_{t+1}=\frac{1}{2}(4[4+1])+$ $\frac{1}{2}(4[4+1])=20, P_{t+1}=-1, E_{t+1}=0$ 


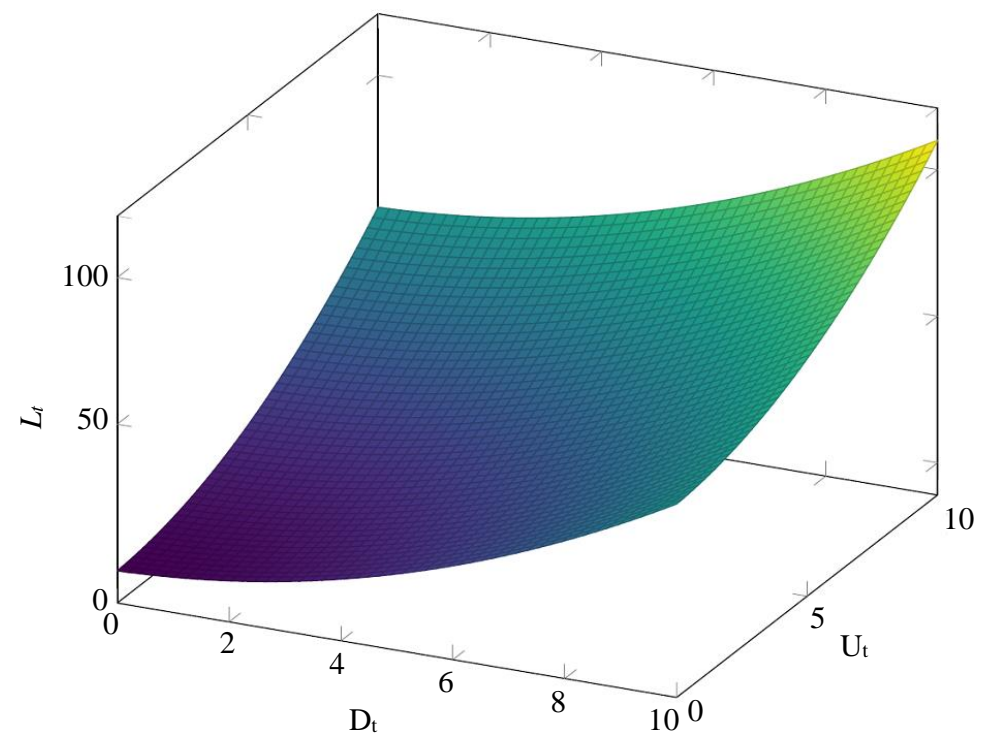

Fig. 6: Drawdown and drawup extremes in BGC stochastic processes the greatest total loss $L_{t}$ occurs when one has the greatest possible $D_{t}$ and $U_{t}$. For the values shown in this surface, this occurs at $D_{t}=10$ and $U_{t}=10$

Substituting (3.8) and (3.9) into (3.7) gives:

$$
\begin{aligned}
& E_{t}=E_{0}+v t-\frac{v}{2}\left[\left(\max _{t \in[0, T]}\left(\sum_{i=0}^{t} \mathfrak{R}_{i}\right)-\sum_{i=0}^{t} \mathfrak{R}_{i}\right)^{2}\right. \\
& +\left(\max _{t \in[0, T]}\left(\sum_{i=0}^{t} \mathfrak{R}_{i}\right)-\sum_{i=0}^{t} \mathfrak{R}_{i}\right) \\
& +\left(\sum_{i=0}^{t} \mathfrak{R}_{i}-\max _{t \in[0, T]}\left(\sum_{i=0}^{t}\left(-\mathfrak{R}_{i}\right)\right)\right)^{2} \\
& \left.+\left(\sum_{i=0}^{t} R_{i}-\max _{t \in[0, T]}\left(\sum_{i=0}^{t}\left(-\mathfrak{R}_{i}\right)\right)\right)\right] \\
& =E_{0}+v t-\frac{v}{2}\left[\left(\max _{t \in[0, T]}\left(\sum_{i=0}^{t} \mathfrak{R}_{i}\right)-\sum_{i=0}^{t} \mathfrak{R}_{i}\right)^{2}\right. \\
& \left.+\left(\sum_{i=0}^{t} \mathfrak{R}_{i}-\max _{t \in[0, T]}\left(\sum_{i=0}^{t} \mathfrak{R}_{i}\right)\right)^{2}\right] \\
& =E_{0}+v t-v\left[\left(\max _{t \in[0, T]}\left(\sum_{i=0}^{t} \mathfrak{R}_{i}\right)\right)^{2}\right. \\
& +2 v\left(\max _{t \in[0, T]}\left(\sum_{i=0}^{t} \mathfrak{R}_{i}\right)\right)\left(\sum_{i=0}^{t} \mathfrak{R}_{i}\right)-v\left(\sum_{i=0}^{t} \mathfrak{R}_{i}\right)^{2},
\end{aligned}
$$

which completes the proof.

This is further illustrated in the following footnote ${ }^{2}$, which agrees with the values in Fig. 5. As will also be seen in the Results section, this formula captures all the

${ }^{2}$ Remark 3.2. From Figure 5a, at $t=18$ one sees that $R_{t}=-2$, $\max _{t \in[0, T]}\left(\sum_{i=0}^{t} R_{i}\right)=3$ and $\min _{t \in[0, T]}\left(\sum_{i=0}^{t} R_{i}\right)=-5$. From (3.7), one sees that $E_{t}=-3 \rightarrow 0$. From Figure $5 \mathrm{~b}$, at $t=19$ one sees that $R_{t}=-1$, $\max _{t \in[0, T]}\left(\sum_{i=0}^{t} R_{i}\right)=3$ and $\min _{t \in[0, T]}\left(\sum_{i=0}^{t} R_{i}\right)=-5$. From (3.7), one sees that $E_{t}=-1 \rightarrow 0$. Notice that $E_{0}=0$ has been set for theoretical purposes, so as to not make the example specific to any particular initial equity and that $E_{t} \leq 0 \rightarrow E=0$. key discrete characteristics of GTP. Before progressing this, recall Doob's Martingale Inequality as it will help simplify our subsequent continuous time version of (3.3).

\section{Lemma 3.3. (Doob's Martingale Inequality)}

Let $X$ be a submartingale taking real values, either in discrete or continuous time. That is, for all times $s$ and $t$ with $s<t$ :

$$
X_{s} \leq \mathbb{E}\left[X_{t} \mid \mathscr{F}_{s}\right]
$$

For a continuous-time submartingale, assume further that the process is cádlág. Then, for any constant $C \in \mathbb{R}_{+}$:

$$
\mathbb{P}\left[\sup _{0 \leq t \leq T} X_{t} \geq C\right] \leq \frac{\mathbb{E}\left[\max \left(X_{T}, 0\right)\right]}{C} .
$$

Let $B$ denote a canonical 1-Dimensional Brownian motion. Then:

$$
\mathbb{P}\left[\sup _{0 \leq t \leq T} B_{t} \geq C\right] \leq \exp \left(-\frac{C^{2}}{2 T}\right) .
$$

Now, to contrast this discrete model by deriving a continuous time Stochastic Differential Equation (SDE) of GTP.

\section{Derivation of $B G C S D E$ of $G T P$}

Theorem 3.4. For a Bi-Directional Grid Constrained Itô diffusion with a given grid width $g \in \mathbb{R}_{+}$, value $v$ per 
grid width, drift $\mu_{t}$ and diffusion $\sigma_{t}$ and Wiener process $W_{t}$ for the rate $R_{t}$, then the change in equity $E_{t}$ over time $t$ is:

$$
\begin{aligned}
& \frac{d E_{t}}{E_{t}}=v t d t-\frac{v}{g}\left[\left(\sup _{t \in[0, T]}\left(\int_{i=0}^{t} R_{i} d t\right)\right)^{2}\right. \\
& \left.-2\left(\sup _{t \in[0, T]}\left(\int_{i=0}^{t} R_{i} d t\right)\right)\left(\int_{i=0}^{t} R_{i} d t\right)+\left(\int_{i=0}^{t} R_{i} d t\right)^{2}\right] \\
& \leq\left[v t+\frac{2 v \mu_{t}}{g}-\frac{v \sigma_{t}^{2}}{g}\right] d t+\left[\frac{2 v \sigma_{t}}{g}\right] d W_{t} .
\end{aligned}
$$

\section{Proof}

In the corresponding continuous time framework $t \in \mathbb{R}_{+} \times \mathbb{R}$ of Fig. 4, one can see that the equity $E_{t}$ at any time $t$ is comprised of the initial equity $E_{0}$, plus the sum of all the winning trades $W_{t}$, minus the sum of all the losing trades $L_{t}$. From (3.1), one can now derive the general formula for $E_{t}$, giving:

$$
\begin{array}{lll}
n=0, & E_{0}=E_{0}, & \\
n=1, & E_{1}=E_{0}+v-v & =E_{0}, \\
n=2, & E_{2}=E_{0}+2 v-3 v & =E_{0}-v, \\
\cdots & \cdots & \cdots \\
n=t, & E_{t}=E_{0}+v t-\underbrace{-v \underbrace{\frac{U_{t}}{2}-v \frac{\left.U_{t}+1\right)}{2}}_{L_{t}\left(D_{t}+1\right)}}_{w_{t}}=E_{0}+v \int_{i=0}^{t} P_{i} d t,
\end{array}
$$

where, $D_{t}$ and $U_{t}$ are the drawdown and drawup of the price $R_{t}$ at time $t$. However, the markets do not trend indefinitely and so $L_{t}$ in (3.12) needs to be replaced with a stochastic process, in this setting, a 1-Dimensional continuous Brownian motion $\int_{i=0}^{t} \mathfrak{R}_{i} d t$ for $\mathfrak{R} \in\{-\alpha, \alpha\}, \forall \alpha \in \mathbb{R}$ giving:

$$
\begin{aligned}
& D_{t}=\sup _{t \in[0, T]}\left(\int_{i=0}^{t} \mathfrak{R}_{i} d t\right)-\mathfrak{R}_{t}, \\
& U_{t}=\mathfrak{R}_{i}-\inf _{t \in[0, T]}\left(\int_{i=0}^{t} \mathfrak{R}_{i} d t\right),
\end{aligned}
$$

In this continuous time stochastic framework, (3.12) scaled down by the count of grid widths $g$ traversed becomes:

$$
\frac{d E_{t}}{E_{t}}=v t d t-\frac{v}{2 g} D_{t}\left(D_{t}+1\right)-\frac{v}{2 g} U_{t}\left(U_{t}+1\right)
$$

where, $E_{t}=E_{0}$ at $t=0$ as an initial condition.

Note that (3.14) is essentially a non-standard Geometric Brownian Motion (GBM). The reason why this was not expressed as an Arithmetic Brownian Motion $(\mathrm{ABM})$ is that the equity random walk $E_{t}$ is required to be modelled as products of random factors and not as sums of random terms. GBM involves independently and identically distributed ratios between successive factors. Furthermore, one requires $\frac{d E_{t}}{E_{t}} \geq 0, \forall t \in \mathbb{R}_{+} \quad$ as trading systems seek to exponentially compound $E$ over time and an $E_{t}=0$ equates to ruin or bankruptcy ${ }^{3}$. In fact, since our $\mu_{t}$ and $\sigma_{t}$ terms are non-constant over time, then our nonstandard GBM is actually a form of the more generalized Itô Processes. Finally, note that (3.14) does not appear at first glance to be a standard GBM as it does not exhibit an explicit $d W_{t}$ term, even though it is implied due to the subsequent use of (3.16).

Substituting (3.13) into (3.14) gives:

$$
\begin{aligned}
& \frac{d E_{t}}{E_{t}}=v t d t-\frac{v}{2 g}\left[D_{t}^{2}+D_{t}\right]-\frac{v}{2 g}\left[U_{t}^{2}+U_{t}\right] \\
& =v t d t-\frac{v}{2 g}\left[\left(\sup _{t \in[0, T]}\left(\int_{i=0}^{t} \Re_{t} d t\right)-\left(\int_{i=0}^{t} \mathfrak{R}_{t} d t\right)\right)^{2}\right. \\
& \left.+\left(\sup _{t \in[0, T]}\left(\int_{i=0}^{t} \Re_{t} d t\right)-\left(\int_{i=0}^{t} \Re_{t} d t\right)\right)\right] \\
& -\frac{v}{2 g}\left[\left(\int_{i=0}^{t} \mathfrak{R}_{t} d t-\inf _{t \in[0, T]}\left(\int_{i=0}^{t} \mathfrak{R}_{t} d t\right)\right)^{2}\right. \\
& \left.+\left(\int_{i=0}^{t} \mathfrak{R}_{t} d t-\inf _{t \in[0, T]}\left(\int_{i=0}^{t} \Re_{t} d t\right)\right)\right] \\
& =v t d t-\frac{v}{2 g}\left(\sup _{t \in[0, T]}\left(\int_{i=0}^{t} \Re_{t} d t\right)-\left(\int_{i=0}^{t} \Re_{t} d t\right)\right)^{2} \\
& -\frac{v}{2 g}\left(\sup _{t \in[0, T]}\left(\int_{i=0}^{t} \Re_{t} d t\right)-\left(\int_{i=0}^{t} \Re_{t} d t\right)\right)-\frac{v}{2 g} \\
& \left(\int_{i=0}^{t} \mathfrak{R}_{t} d t--\sup _{t \in[0, T]}\left(\int_{i=0}^{t}-\mathfrak{R}_{t} d t\right)\right)^{2} \\
& -\frac{v}{2 g}\left(\int_{i=0}^{t} \Re_{t} d t--\sup _{t \in[0, T]}\left(\int_{i=0}^{t}-\Re_{t} d t\right)\right) \\
& =v t d t-\frac{v}{2 g}\left(\sup _{t \in[0, T]}\left(\int_{i=0}^{t} \Re_{t} d t\right)-\left(\int_{i=0}^{t} \Re_{t} d t\right)\right)^{2} \\
& -\frac{v}{2 g}\left(\int_{i=0}^{t} \Re_{t} d t-\sup _{t \in[0, T]}\left(\int_{i=0}^{t} \Re_{t} d t\right)\right)^{2} \\
& =v t d t-\frac{v}{g}\left[\left(\sup _{t \in[0, T]}\left(\int_{i=0}^{t} \mathfrak{R}_{t} d t\right)\right)^{2}\right. \\
& -2\left(\sup _{t \in[0, T]}\left(\int_{i=0}^{t} \Re_{t} d t\right)\left(\int_{i=0}^{t} \Re_{t} d t\right)\right) \\
& \left.+\left(\int_{i=0}^{t} \Re_{t} d t\right)^{2}\right] \text {. }
\end{aligned}
$$

One can now formalize this continuous Brownian motion by adopting the simplest of 1-Dimensional Itô Diffusion processes, where (3.5) expands to:

${ }^{3}$ The fact that there are rare cases, where one can loose more than 0 , i.e., $E_{t}<0$, due to the Broker and/or Trader not closing down enough losing trades during a margin call, means that such scenarios will be treated mathematically as if there is an Absorption Barrier (Kac, 1945) at $E_{t}=0$ without any loss of generality. 


$$
R_{t}=\int_{i=0}^{t} \Re_{i} d t:=\mu_{t} d t+\sigma_{t} d W_{t} .
$$

One now applies Doob's Martingale Inequality together with the Itô Isometry:

$$
\mathbb{E}\left[\left(\int_{0}^{T} X_{t} d W_{t}\right)^{2}\right]=\mathbb{E}\left[\int_{0}^{T} X_{t}^{2} d t\right]
$$

and (3.16) to simplify (3.15) into:

$$
\begin{aligned}
& \frac{d E_{t}}{E_{t}} \leq v t d t-\frac{v}{g}\left[\left(\exp \left(-\frac{\lambda^{2}}{2 t}\right)\right)^{2}-2\left(\exp \left(-\frac{\lambda^{2}}{2 t}\right)\right)\left(\int_{i=0}^{t} \Re_{t} d t\right)\right. \\
& \left.+\left(\int_{i=0}^{t} \Re_{t} d t\right)^{2}\right] \\
& =v t d t-\frac{v}{g}\left(\exp \left(-\frac{\lambda^{2}}{2 t}\right)\right)^{2}-\frac{2 v}{g} \exp \left(-\frac{\lambda^{2}}{2 t}\right)\left(\mu_{t} d t+\sigma_{t} d W_{t}\right) \\
& -\frac{v}{g}\left(\mu_{t}^{2}(d t)^{2}+2 \sigma_{t} \mu_{t} d t d W_{t}+\sigma_{t}^{2}\left(d W_{t}\right)^{2}\right) .
\end{aligned}
$$

It is well known (Øksendal, 1995; Shreve, 2004) that in the limit $d t \rightarrow 0$, the terms $(d t)^{2}$ and $d t d W_{t}$ tend to zero faster than $\left(d W_{t}\right)^{2}$, which is $O(d t)$. Setting the $(d t)^{2}$ and $d t d W_{t}$ terms to zero, substituting $d t$ for $\left(d W_{t}\right)^{2}$ (due to the quadratic variance of a Wiener process) and collecting the $d t$ and $d W_{t}$ terms, one obtains from (3.17):

$$
\begin{aligned}
& \frac{d E_{t}}{E_{t}} \leq v t d t-\frac{v}{g}\left(\exp \left(-\frac{\lambda^{2}}{2 t}\right)\right)^{2}-\frac{2 v}{g} \exp \left(-\frac{\lambda^{2}}{2 t}\right)\left(\mu_{t} d t+\sigma_{t} d W_{t}\right) \\
& -\frac{v}{g}\left(\sigma_{t}^{2} d t\right) \\
& =-\frac{v}{g}\left(\exp \left(-\frac{\lambda^{2}}{2 t}\right)\right)^{2}+\left[v t+\frac{2 v \mu_{t}}{g} \exp \left(-\frac{\lambda^{2}}{2 t}\right)-\frac{v \sigma_{t}^{2}}{g}\right] d t \\
& +\left[\frac{2 v \sigma_{t}}{g} \exp \left(-\frac{\lambda^{2}}{2 t}\right)\right] d W_{t} .
\end{aligned}
$$

However, most SDEs, especially nonlinear SDEs, do not have analytical solutions and so one has to resort to numerical approximation schemes in order to simulate sample paths of solutions to the given equation. Also note that the term $-\frac{v}{g} \ll+\infty$, is constant over $d t$ and $d W_{t}$ and is eclipsed (become less significant) by the variable terms over time. This together with the observation that:

$$
\lim _{t \rightarrow+\infty}\left\{\left[\exp \left(-\frac{\lambda^{2}}{2 t}\right)\right]^{2}\right\}=1
$$

simplifies (3.18) to:

$$
\begin{aligned}
& \frac{d E^{t}}{E_{t}} \leq\left[v t+\frac{2 v \mu_{t}}{g}-\frac{v \sigma_{t}^{2}}{g}\right] d t+\left[\frac{2 v \sigma_{t}}{g}\right] d W_{t} \\
& =\Gamma_{1} d t+\Gamma_{2} d W_{t},
\end{aligned}
$$

where, $\Gamma_{1}=v t+\frac{2 v \mu_{t}}{g}-\frac{v \sigma_{t}^{2}}{g}$ and $\Gamma_{2}=\frac{2 v \sigma_{t}}{g}$, completing the proof.

It is worthwhile noting at this stage, setting aside the constants $v$ and $g$, that since $\Gamma_{1}\left(t, \mu t, \sigma_{t}\right)$ and $\Gamma_{2}\left(\sigma_{t}\right)$, then (3.19) is not a standard simple linear SDE and that there is some convolution of $\sigma_{t}^{2}$ within the deterministic component $d_{t}$ with the $\sigma_{t}$ within the random component $d W_{t}$. This means that one would expect to see some relatively complex interactions from the underlying distribution samples. For example, negative $\sigma_{t}$ values becoming positive due to $\sigma_{t}^{2}$, skewing the results towards $E_{t} \rightarrow 0$ due to the negative sign before $\sigma_{t}^{2}$, which supports to a certain extent why $E_{t}$ has a tendency to almost surely approach 0 over time (subject to certain drift and diffusion conditions set out in the Results section).

\section{Solution of SDE of GTP}

\section{Theorem 3.5}

For a Bi-Directional Grid Constrained Brownian motion with a given grid width $g$, value $v$ per grid width, drift $\mu_{t}$, diffusion $\sigma_{t}$ and Wiener process $W_{t}$ for the rate $R_{t}$, then the equity $E_{t}$ over time $t$ has the solution:

$$
E_{t}=E_{0} \exp \left(\left[v t+\frac{2 v \mu_{t}}{g}-\frac{v \sigma_{t}^{2}}{g}-\frac{2 v^{2} \sigma_{t}^{2}}{g^{2}}\right] t+\left[\frac{2 v \sigma_{t}}{g}\right] W_{t}\right)
$$

\section{Proof}

Recall that (3.18) is a GBM whose well known (Øksendal, 1995; Shreve, 2004) general solution is of the form:

$$
\begin{aligned}
& \int_{0}^{t} d \ln \left(S_{s}\right)=\int_{0}^{t}\left(\mu_{s}-\frac{\sigma_{s}^{2}}{2}\right) d s+\int_{0}^{t} \sigma_{s} d W_{s} \\
& \ln \left(S_{t}\right)-\ln \left(S_{0}\right)=\left(\mu_{t}-\frac{\sigma_{t}^{2}}{2}\right) t+\sigma_{t} W_{t}
\end{aligned}
$$

$\exp \left(\ln \left(\frac{S_{t}}{S_{0}}\right)\right)=\exp \left(\left(\mu_{t}-\frac{\sigma_{t}^{2}}{2}\right) t+\sigma_{t} W_{t}\right)$

$S_{t}=S_{0} \exp \left(\left(\mu_{t}-\frac{\sigma_{t}^{2}}{2}\right) t+\sigma_{t} W_{t}\right)$. 
One is now in a position to solve the Bi-Directional Grid Constrained SDE (3.19) by substituting $\Gamma_{1}$ and $\Gamma_{2}$. However, due to the $t$ term in $\Gamma_{1}$, one needs to do the substitution into (3.20) rather than in (3.21) and also make use of a change of variable $s$ :

$$
\begin{aligned}
& \int_{0}^{t} d \ln \left(E_{s}\right)=\int_{0}^{t}\left(\Gamma_{1}-\frac{1}{2} \Gamma_{2}^{2}\right) d s+\int_{0}^{t} \Gamma_{2} d W_{s} \\
& =\int_{0}^{t}\left(\left[v s+\frac{2 v \mu_{s}}{g}-\frac{v \sigma_{s}^{2}}{g}\right]-\frac{1}{2}\left[\frac{2 v \sigma_{s}}{g}\right]^{2}\right) d s+\int_{0}^{t}\left[\frac{2 v \sigma_{s}}{g}\right] d W_{s} \\
& =v \int_{0}^{t} s d s+\frac{2 v \mu_{s}}{g} \int_{0}^{t} d s-\frac{v \sigma_{s}^{2}}{g} \int_{0}^{t} d s-\frac{2 v^{2} \sigma_{s}^{2}}{g^{2}} \int_{0}^{t} d s \\
& +\frac{2 v \sigma_{s}}{g} \int_{0}^{t} d W_{s} .
\end{aligned}
$$

By integrating, one obtains:

$$
\begin{aligned}
& \int_{0}^{t} d \ln \left(E_{s}\right)=\left[v t+\frac{2 v \mu_{t}}{g}-\frac{v \sigma_{t}^{2}}{g}-\frac{2 v^{2} \sigma_{t}^{2}}{g^{2}}\right] t+\left[\frac{2 v \sigma_{t}}{g}\right] W_{t} \\
& \ln \left(E_{t}\right)-\ln \left(E_{0}\right)=\left[v t+\frac{2 v^{2} \mu_{t}}{g^{2}}-\frac{v^{2} \sigma_{t}^{2}}{g^{2}}-\frac{2 v^{4} \sigma_{t}^{2}}{g^{4}}\right] t+\left[\frac{2 v^{2} \sigma_{t}}{g^{2}}\right] W_{t} \\
& \exp \left(\ln \left(\frac{E_{t}}{E_{0}}\right)\right)=\exp \left(\left[v t+\frac{2 v \mu_{t}}{g}-\frac{v \sigma_{t}^{2}}{g}-\frac{2 v^{2} \sigma_{t}^{2}}{g^{2}}\right] t+\left[\frac{2 v \sigma_{t}}{g}\right] W_{t}\right) \\
& \therefore E_{t}=E_{0} \exp \left(\left[v t+\frac{2 v \mu_{t}}{g}-\frac{v \sigma_{t}^{2}}{g}-\frac{2 v^{2} \sigma_{t}^{2}}{g^{2}}\right] t+\left[\frac{2 v \sigma_{t}}{g}\right] W_{t}\right),
\end{aligned}
$$

which completes the proof.

\section{Mean and Variance of SDE of GTP}

It is worthwhile noting that the interrelationship between drift and diffusion will determine $E_{t}$, as shown in Fig. 7.

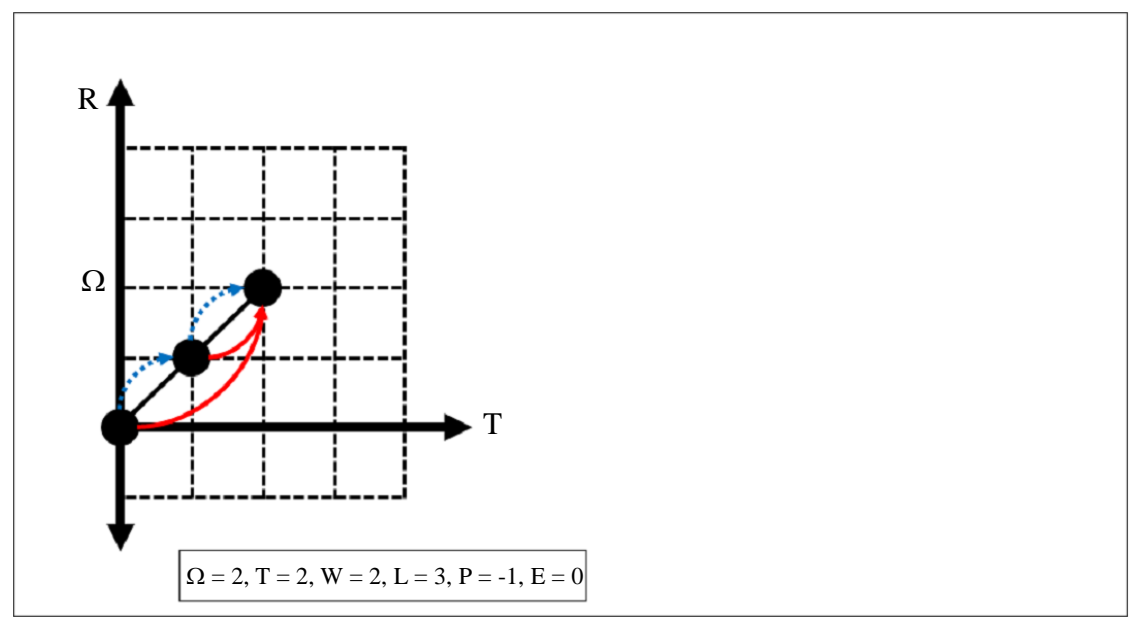

(a)

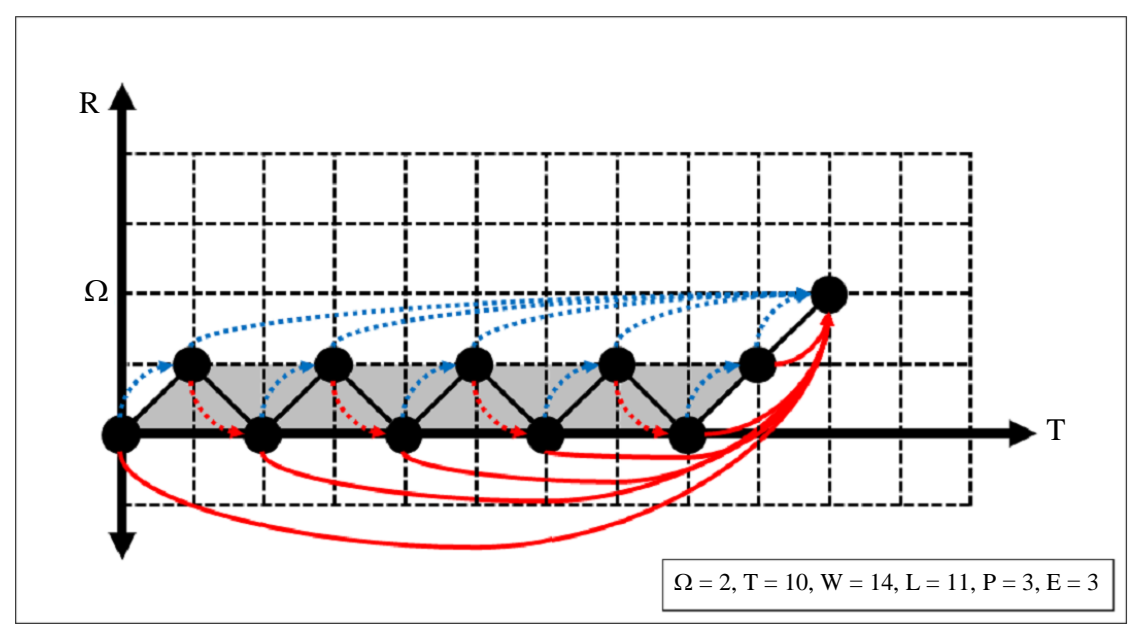

(b)

Fig. 7: Expected Value and Variance for Rate Random Walk $R_{t}$ and Bi-Directional Grid Trading Equity $E_{t}$ for a $\Omega$ (a). $\Omega$ is reached in the quickest time and corresponding Profit is the lowest. (b). $\Omega$ is reached over a longer time and the corresponding Profit is not as low 
One can now elaborate this further by deriving the expected value $\mathbb{E}\left[E_{t}\right]$ and variance $\mathbb{V}\left[E_{t}\right]$ :

$$
\begin{aligned}
& \mathbb{E}\left[E_{t}\right]=\mathbb{E}\left[E_{0} \exp \left(\Gamma_{1} t+\Gamma_{2} W_{t}\right)\right] \\
& =E_{0} \exp \left(\Gamma_{1} t\right) \times \mathbb{E}\left[\exp \left(\Gamma_{2} W_{t}\right)\right] \\
& =E_{0} \exp \left(\left[v t+\frac{2 v \mu_{t}}{g}-\frac{v \sigma_{t}^{2}}{g}-\frac{2 v^{2} \sigma_{t}^{2}}{g^{2}}\right] t\right) \\
& \times \mathbb{E}\left[\exp \left(\left[\frac{2 v \sigma_{t}}{g}\right] W_{s}\right)\right] .
\end{aligned}
$$

Now let $X \sim \mathscr{N}(0,1)$ and $a \in \mathbb{R}$ in the following generalized Gaussian expectation:

$$
\begin{aligned}
& \mathbb{E}[\exp (a X)]=\int_{\mathbb{R}} \frac{1}{\sqrt{2 \pi}} \exp \left(-\frac{1}{2} x^{2}\right) \exp (a x) d x \\
& =\int_{\mathbb{R}} \frac{1}{\sqrt{2 \pi}} \exp \left(-\frac{1}{2}(x-a)^{2}+\frac{1}{2} a^{2}\right) \\
& =\exp \left(\frac{1}{2} a^{2}\right) \int_{\mathbb{R}} \frac{1}{\sqrt{2 \pi}} \exp \left(-\frac{1}{2}(x-a)^{2}\right) \\
& =\exp \left(\frac{1}{2} a^{2}\right)
\end{aligned}
$$

because $x \mapsto \frac{1}{\sqrt{2 \pi}} \exp \left(-\frac{1}{2}(x-a)^{2}\right)$ is the density of a $\mathscr{N}(a, 1)$ distribution. Hence:

$$
\mathbb{E}\left[\exp \left(\left[\frac{2 v \sigma_{t}}{g}\right] W_{t}\right)\right]=\exp \left(\frac{1}{2}\left[\frac{2 v \sigma_{t}}{g}\right]^{2}\right)=\exp \left(\frac{2 v^{2} \sigma_{t}^{2}}{g^{2}}\right) .
$$

Now (3.23) can be expanded:

$$
\begin{aligned}
& \mathbb{E}\left[E_{t}\right]=E_{0} \exp \left(\left[v t+\frac{2 v \mu_{t}}{g}-\frac{v \sigma_{t}^{2}}{g}-\frac{2 v^{2} \sigma_{t}^{2}}{g^{2}}\right] t\right) \exp \left(\frac{2 v^{2} \sigma_{t}^{2}}{g^{2}}\right) \\
& =E_{0} \exp \left(\left[v t+\frac{2 v \mu_{t}}{g}-\frac{v \sigma_{t}^{2}}{g}-\frac{2 v^{2} \sigma_{t}^{2}}{g^{2}}\right] t+\frac{2 v^{2} \sigma_{t}^{2}}{g^{2}}\right) .
\end{aligned}
$$

By using the exact same approach, one also finds that the variance of $E_{t}$, i.e., $\mathbb{V}\left(E_{t}\right)$ is derived as follows, noting our definitions of $\Gamma_{1}$ and $\Gamma_{2}$ :

$$
\begin{aligned}
& \mathbb{V}\left(E_{t}\right)=E_{0} \exp \left(2 \Gamma_{1} t\right)\left[\exp \left(\Gamma_{2}^{2} t\right)-1\right] \\
& =E_{0}^{2} \exp \left(2\left[v t+\frac{2 v \mu_{t}}{g}-\frac{v \sigma_{t}^{2}}{g}-\frac{2 v^{2} \sigma_{t}^{2}}{g^{2}}\right] t\right) \\
& \times\left[\exp \left(\left(\frac{2 v \sigma_{t}}{g}\right)^{2} t\right)-1\right] \\
& =E_{0}^{2} e^{\left(2 v t+\frac{4 v \mu_{t}-2 v \sigma_{t}^{2}}{g}-\frac{4 v^{2} \sigma_{t}^{2}}{g^{2}}\right) t}\left(e^{\left(\frac{4 v^{2} \sigma_{t}^{2}}{g^{2}}\right) t}-1\right) .
\end{aligned}
$$

Note that this preserves the known property that the variance of GBM starts at 0 and then grows exponentially. This means that the swings up and down become larger and larger over time and further supports why grid trading systems can suddenly lead to ruin or bankruptcy.

\section{Results and Discussion}

Having derived both a DDE model and a SDE model for the GTP, one can now revert to the numerical methods of Monte Carlo Simulation and Brute Force Combinatorial Enumeration to complement the derived theoretical framework.

\section{Simulation of BGC DDE of GTP}

The simulation of the discrete model is shown in Fig. 8.

From Fig. 8, one sees that $R_{t}$ trending for too long in one direction leads to large losses in $E_{t}$, whereas $R_{t}$ range bound (non-trending) leads to large gains in $E_{t}$. This further highlights the sensitivity of $E_{t}$ to small changes in $R_{t}$. The key benefit of the discrete model is that fund managers and investors alike can anticipate the growth and decline in $E_{t}$ in relation to the underlying $R_{t}$ by monitoring $D_{t}$ and $U_{t}$ and take then various money management measures and strategies to maximize $E_{t}$.

To further help visualize the DDE theorem of the GTP, one can now simulate the discrete distribution for $R_{t}$ for $t \in[0,20]$ using brute force combinatorial enumeration. Since $\mathfrak{R}_{i} \in\{-1,1\}$, there are $2^{20}=$ $1,048,576$ possible paths to simulate. To expand this over a greater time period would not reveal any possible additional 'hidden' distribution properties and so the comprehensive distribution is shown in Fig. 9.

Figure 9 shows the typical distribution of a standard diffusion process for a discrete random walk, albeit over a binomial lattice model, hence the 'holes' on the surface. It is understood that as the lattice mesh width becomes infinitesimally smaller, then this distribution approaches the Gaussian distribution and that these 'holes' disappear.

By implementing (3.7), one is able to Fig. 10, the constraining impact of the Bi-Directional Grid Constraining on $E_{t}$ from the underlying $R_{t}$ distribution.

Figure 10 shows that as time increases, there are many more profitable trades that occur, resulting in a greater accumulation of positive profit $P_{t} \in \mathbb{Z}_{+}$. What is not so apparent is that there are numerous smaller occurrences of very negative profits $P_{t} \in \mathbb{Z}_{-}$that can, at times, outweigh the positive profits and lead to ruin. Also note that these ruin events accumulate in smaller parallel distributions alongside the main positive distribution density. 

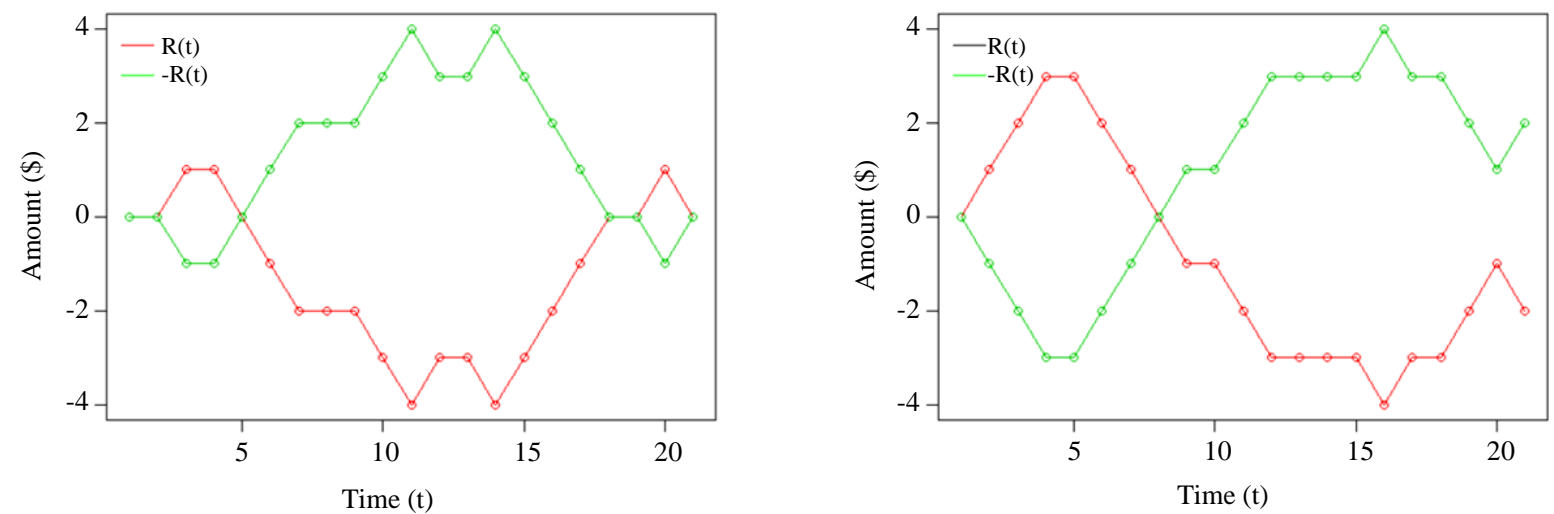

(a). Scenario 1 (d). Scenario 2

$R_{t},-R_{t}$
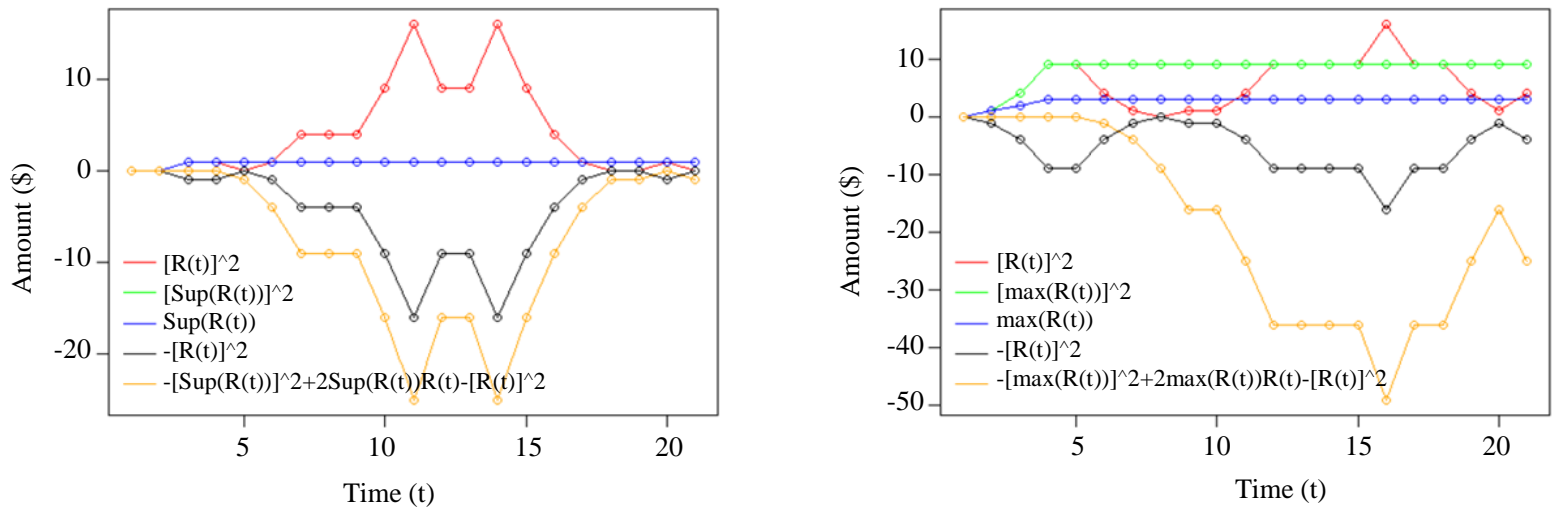

(b). Scenario 1 (e). Scenario 2

$(\sup [B(t)])^{2},[B(t)]^{2},-(\sup [B(t)])^{2}+2 B(t) \sup [B(t)]-[B(t)]^{2}$
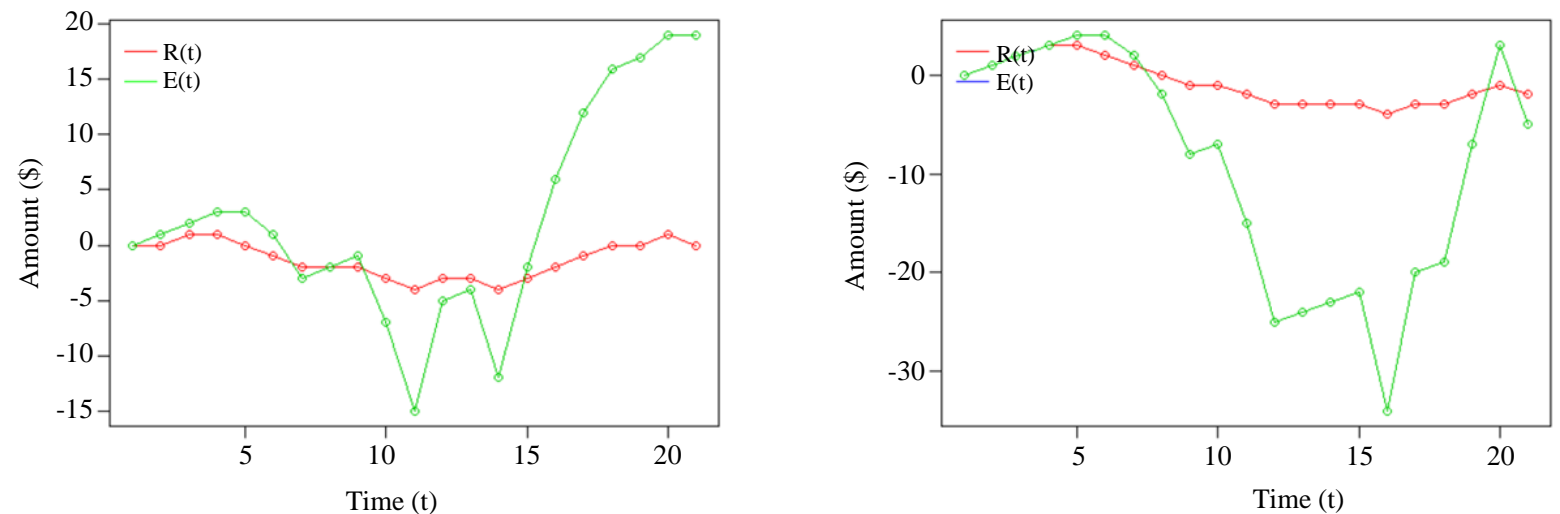

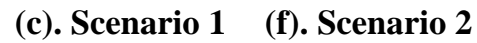

$B(t), E(t)$

Fig. 8: Simulations of Discrete Difference Equation (DDE) of GTP; (a). shows $R_{t}$ and $-R_{t}$, which drives (b). containing the underlying components that constrain $E_{t}$ in (c). Scenario 2: (d). shows a different $R_{t}$ and $-R_{t}$, which drives (e). containing the underlying components that constrain $E_{t}$ in (f). Notice that the model for $E_{t}$ is sensitive to the underlying $R_{t}$ volatility changes captured by $D_{t}$ and $U_{t}$ 


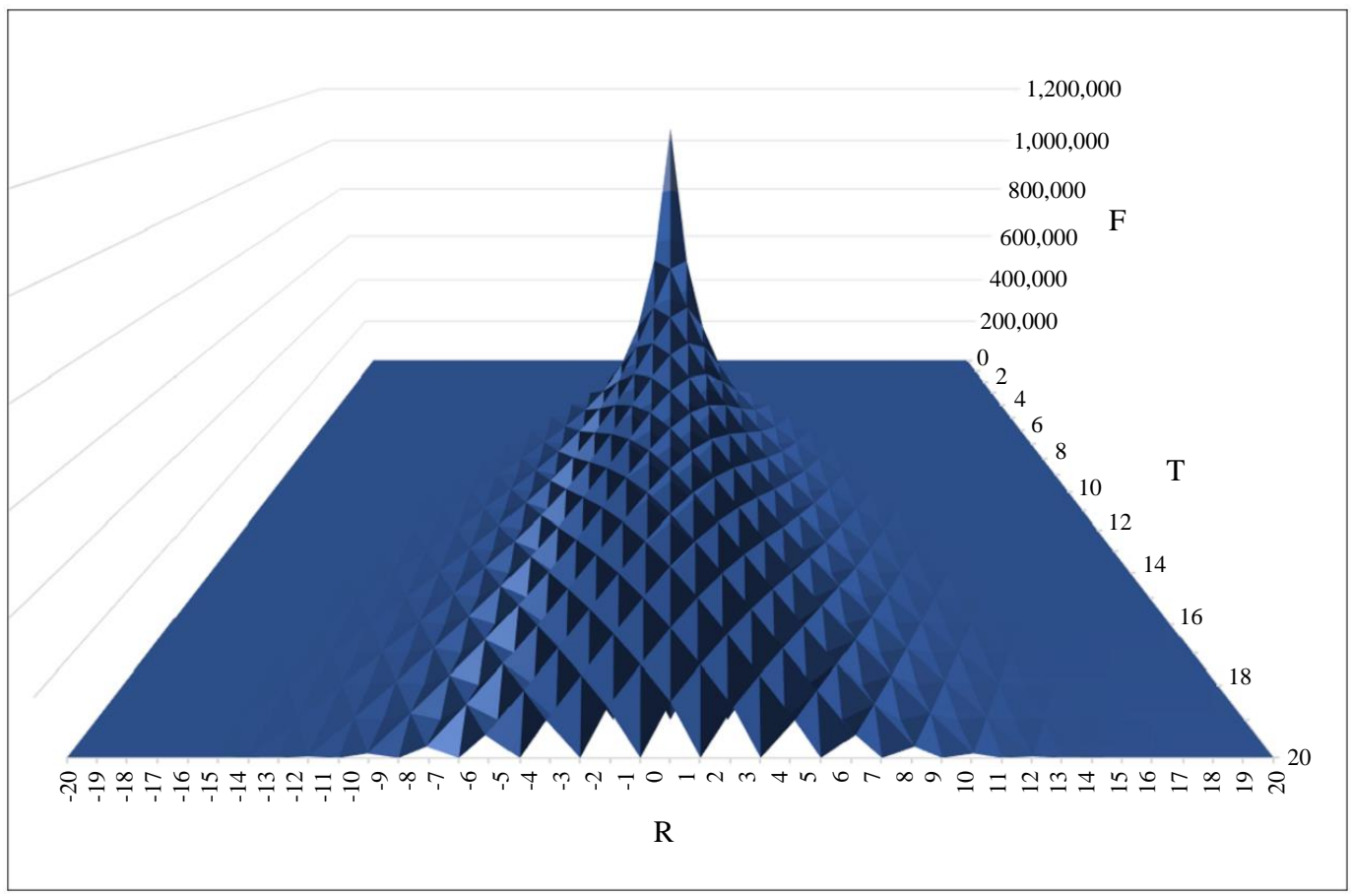

Fig. 9: Distribution of Rate $R_{t}$ over time $T$ Since $R_{t}$ is discrete, so too is $F\left(R_{t}\right)$. Notice that the distribution has 'holes' since the discrete binomial lattice model does not permit certain paths reaching certain points (such as at $t=1, R_{t} \neq 0$ as $\mathfrak{R}_{i} \in\{-1,1\}$ )

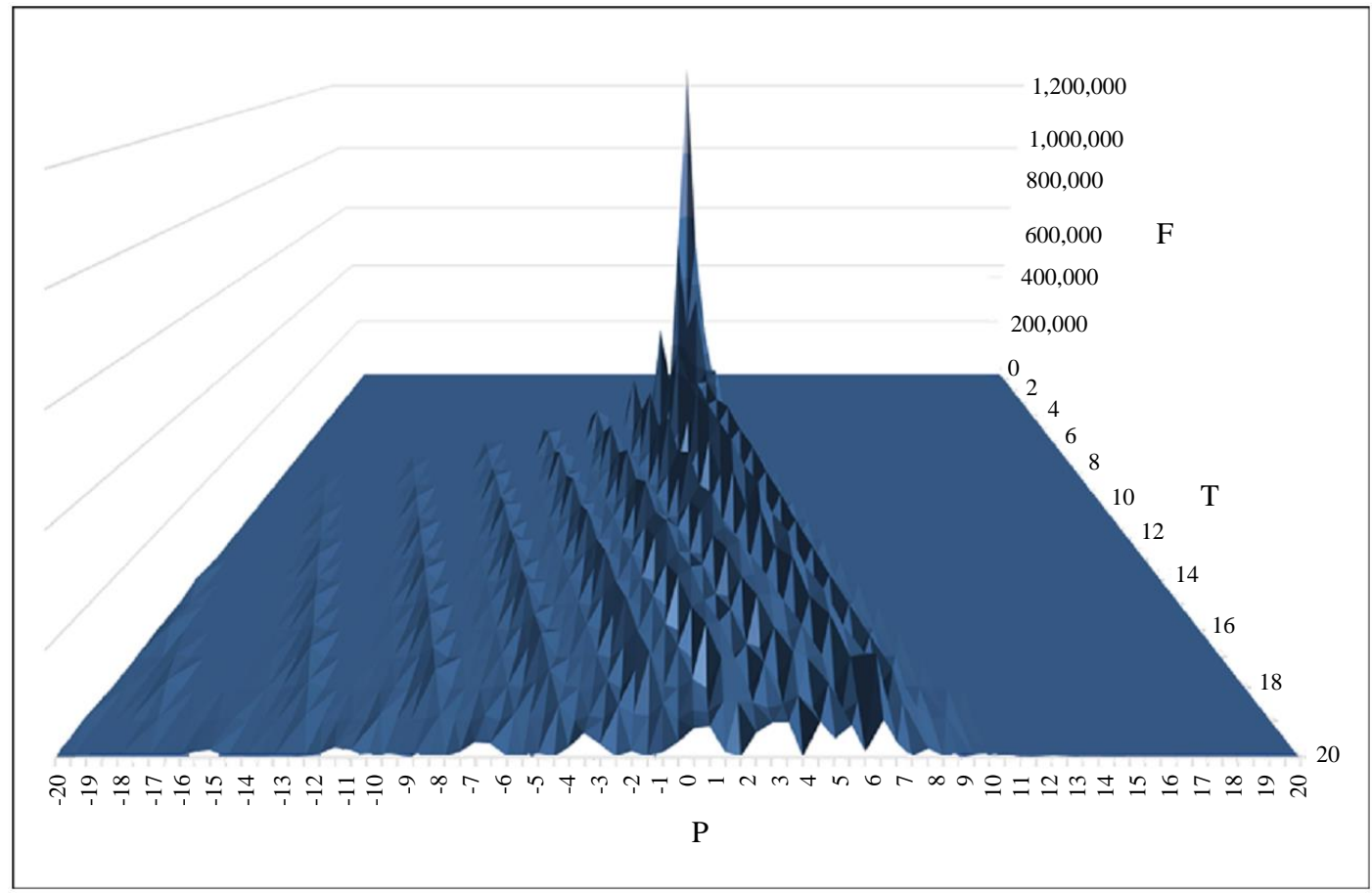

Fig. 10: Distribution of Profit $P_{t}$ over time $T$ Since $P_{t}$ is discrete, so too is $F\left(P_{t}\right)$. The distribution of $P_{t}$ shows that grid trading provides many opportunities to achieve positive profits but fewer opportunities that result in much more severe negative profits (i.e., losses) that can lead to ruin. The distribution is also partitioned into sub-distributions or accumulation zones with certain profit paths and corresponding densities do not exist (either not at all or existing but statistically insignificant) 


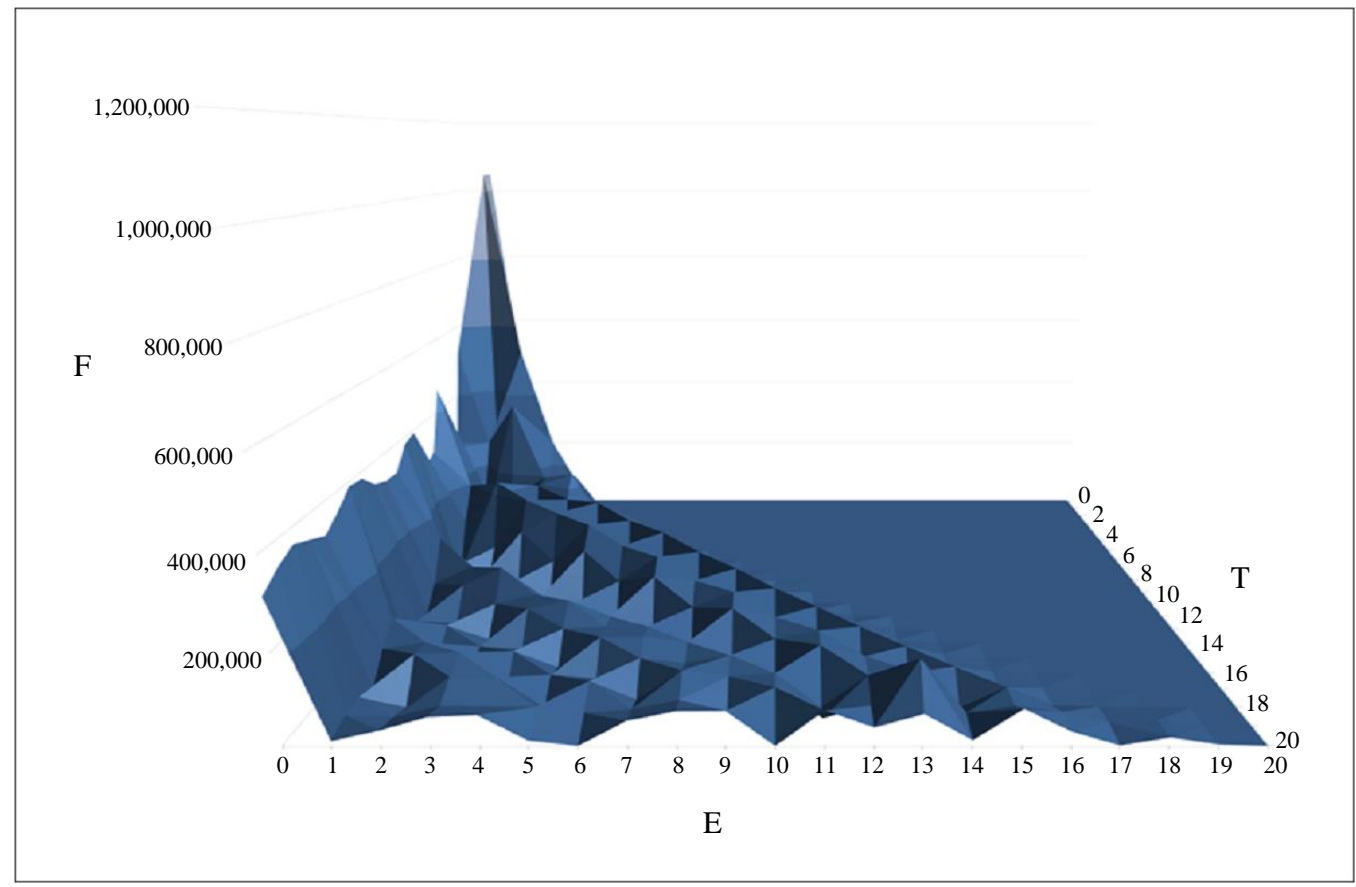

Fig. 11: Distribution of Equity $E_{t}$ over time $T$ Since $E_{t}$ is discrete, so too is $F\left(E_{t}\right)$

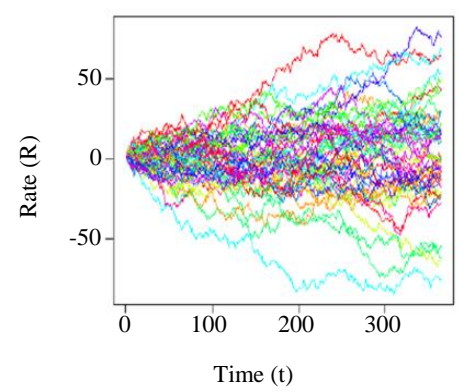

(a). Scenario 1 of $\boldsymbol{R}_{t}$

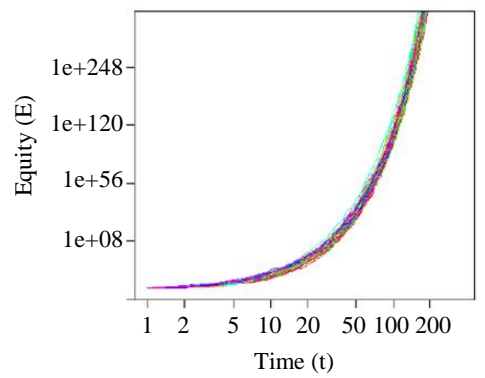

(b). Scenario 1 of $E_{t}$

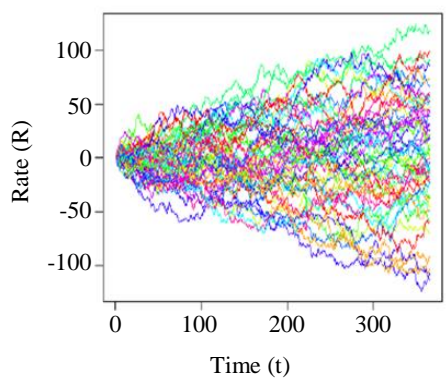

(c). Scenario 2 of $R_{t}$

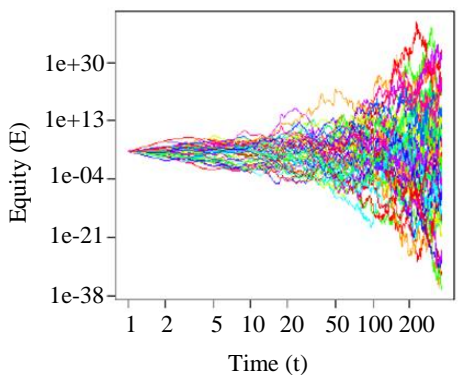

(d). Scenario 2 of $E_{t}$

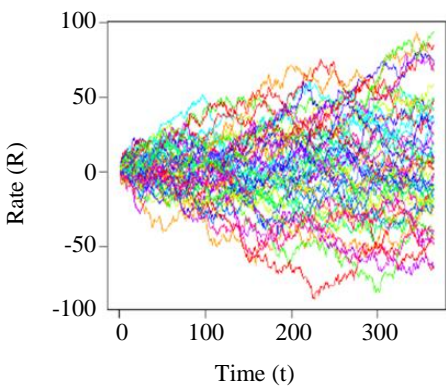

(e). Scenario 3 of $R_{t}$

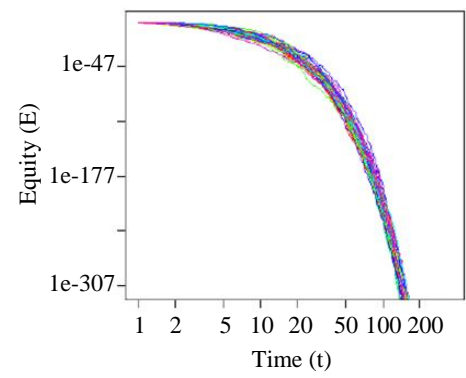

(f). Scenario 3 of $E_{t}$

Fig. 12: Simulations of Continuous SDE of GTP The simulations for Scenario 1, 2 and 3 (in (a)., (c)., (e). respectively) all look quite similar to the 'naked eye' and indeed have very similar values of $\sigma_{t}$ (namely 30, 35 and 40) whilst all other parameters $(v, g$, $\mu_{t}$ ) were kept constant. However, the sensitivity of $E_{t}$ to initial conditions is amplified due to the constraining nature of how $E_{t}\left(R_{t}\right)$ is a function of $R_{t}$. The variance $\sigma_{t}$ was the most sensitive parameter resulting from the sensitivity analysis and shows how increasing it from 30 to 40 can transition $E_{t}$ from (b). 100\% highly profitable paths, (d). 50\% profitable and 50\% losing paths, (f). $100 \%$ highly losing paths 
Finally, note that the equity $E_{t}$ acts as an absorption barrier at $P_{t}=0$ since $P_{t} \leq 0 \rightarrow E_{t}=0$, as shown in Fig. 11 .

From Fig. 11, the absorption barrier at $P_{t}=0$ effectively maps the distribution of $R_{t}: t \in(-\infty, 0) \rightarrow E_{t}$ $=0$, increasing the accumulation at $E_{t}=0$. Figure 11 should thus be compared with Fig. 10 and further supports the fact that the GTP provides many opportunities to generate positive profits, but also provides fewer opportunities to wipe out any possible gain and indeed one's initial capital $E_{0}$.

\section{Simulation of BGC SDE of GTP}

To complement these discrete results, one can simulate numerous sample paths for various parameters of the continuous model in Fig. 12.

From Fig. 12, notice that the three $R_{t}$ scenarios all look similar, however, the resulting Bi-Directional Grid Constrained SDE of $E_{t}$ shows that it is sensitive to initial conditions $\left(\sigma_{t}\right.$ moreso than $\left.\mu_{t}\right)$. Sensitivity analysis of $v$ and $g$ showed only a minor impact in comparison.

\section{Conclusion}

This paper has extended the previous research on Maximal Drawdown for Long-only trading and investment strategies such as shares and extended the research by incorporating the Drawdown and Drawup for Long and Short (Bi-Directional) Grid Constrained (BGC) trading and investment strategies such as Foreign Exchange (FX) and other types of derivative instruments. Both the discrete properties of random walks and the continuous properties of Itô diffusion (collectively stochastic processes) were examined as they traverse a BGC binomial lattice model. Novel theorems for a Discrete Difference Equation (DDE) and a continuous Stochastic Differential Equation (SDE) model of $E_{t}$ were derived and proved for the Grid Trading Problem (GTP).

This constrained environment forms a rich framework to further study such stochastic processes in their own right, but can also lead to further applications in quantitative finance, funds management, investment analysis and banking risk management. In particular, this can lead to optimized risk management of hedging and optimized profit growth strategies.

\section{Acknowledgment}

We would like to thank Prof. Martin Schweizer of ETH Züurich and A/Prof. Ron Addie of University of Southern Queensland for their invaluable advice on refining this paper. We would also like to thank the independent referees of this journal for their endorsements and suggestions.

\section{Funding Information}

The first author was supported by an Australian Government Research Training Program (RTP) Scholarship.

\section{Author's Contributions}

Aldo Taranto: Conceptualization, methodology, software, investigation, writing - original draft, writing review and editing, formal analysis and visualization.

Shahjahan Khan: Validation and supervision.

\section{Ethics}

This article is original and contains unpublished material. The corresponding author confirms that all of the other authors have read and approved the manuscript and that there are no ethical issues involved.

\section{References}

AdmiralMarkets. (2017). Forex grid trading strategy explained.

Biagini, F., Guasoni, P., \& Pratelli, M. (2000). Mean-variance hedging for stochastic volatility models. Mathematical Finance, 10(2), 109-123.

Černý, A., \& Kallsen, J. (2007). On the structure of general mean-variance hedging strategies. The Annals of probability, 35(4), 1479-1531.

Czichowsky, C., Muhle-Karbe, J., \& Schachermayer, W. (2012). Transaction costs, shadow prices and connections to duality. preprint, 1205.

Davis, M. H., \& Norman, A. R. (1990). Portfolio selection with transaction costs. Mathematics of operations research, 15(4), 676-713.

Duffie, D., \& Richardson, H. R. (1991). Mean-variance hedging in continuous time. The Annals of Applied Probability, 1(1), 1-15.

Dumas, B., \& Luciano, E. (1991). An exact solution to a dynamic portfolio choice problem under transactions costs. The Journal of Finance, 46(2), 577-595.

DuPloy, A. (2008). The expert4x, no stop, hedged, grid trading system and the hedged, multi-currency, forex trading system.

DuPloy, A. (2010). Expertgrid expert advisor user's guide.

Feller, W. (1951). The asymptotic distribution of the range of sums of independent random variables. The Annals of Mathematical Statistics, 427-432.

Fleming, W. H., \& Hernández-Hernández, D. (2003). An optimal consumption model with stochastic volatility. Finance and Stochastics, 7(2), 245-262.

Graversen, S., \& Shiryaev, A. (2000). An extension of p. Levy's distributional properties to the case of a Brownian motion with drift. Bernoulli, 6(4), 615-620. 
Guasoni, P., \& Muhle-Karbe, J. (2013). Portfolio choice with transaction costs: a user's guide. In ParisPrinceton Lectures on Mathematical Finance 2013 (pp. 169-201). Springer, Cham.

Haigh, M. S., \& Holt, M. T. (2000). Hedging multiple price uncertainty in international grain trade. American Journal of Agricultural Economics, 82(4), 881-896.

Harris, M. (1998). Grid hedging currency trading.

Hu, Y., Shi, Z., \& Yor, M. (2015). The maximal drawdown of the Brownian meander. Electronic Communications in Probability, 20.

Kac, M. (1945). Random walk in the presence of absorbing barriers. The Annals of Mathematical Statistics, 16(1), 62-67.

King, J. (2010). ForexGridMaster v3.01 manual. https://moam.info/forexgridmaster-v301-manualforexgridmastercomforex_59c762301723ddf980311864.html

King, J. (2015). ForexGridMaster 5.1 advanced manual. https://www.forexgridmaster.com/download/forexgr idmaster-advanced-v5-2-manual/

Magdon-Ismail, M., Atiya, A., Pratap, A., \& AbuMostafa, Y. (2004). On the maximum drawdown of a brownian motion. Journal of Applied Probability, 41(1).

Mitchell, C. (2018). Grid trading. Retrieved from https://www.investopedia.com/terms/g/gridtrading.asp

Merton, R. (1971). Optimal consumption and portfolio rules in a continuous-time model. Journal of Economic Theory, 3, 373-413.

Muhle-Karbe, J., \& Liu, R. (2012). Portfolio selection with small transaction costs and binding portfolio constraints. SIAM Journal on Financial Mathematics, 4(1), 203-227.

Neal, D. K. (2013). Average extrema of a random walk with a negative binomial stopping time. International Journal of Pure and Applied Mathematics, 88(3), $391-405$.
Øksendal, B. (1995). Stochastic Differential Equations: An Introduction with Applications. Springer, New York.

Rogers, L., \& Stapleton, E. (2002). Utility maximisation with time-lagged trading. Computational Methods in Decision-Making, Economics and Finance, 249-269.

Schweizer, M. (2010). Mean-variance hedging. Encyclopedia of quantitative finance.

Shreve, S. E. (2004). Stochastic calculus for finance II: Continuous-time models (Vol. 11). Springer Science \& Business Media.

Stulz, R. (2013). How companies can use hedging to create shareholder value. Journal of Applied Corporate Finance, 25(4), 21-29.

Taranto, A., \& Khan, S. (2020a). Bi-directional grid absorption barrier constrained stochastic processes with applications in finance and investment. Risk Governance and Control: Financial Markets and Institutions, 10(3), 20-33.

Taranto, A., \& Khan, S. (2020b). Gambler's ruin problem and bi-directional grid constrained trading and investment strategies. Investment Management and Financial Innovations, 17(3), 54-66.

Thomson, R. J. (2005). The pricing of liabilities in an incomplete market using dynamic mean-variance hedging. Insurance: Mathematics and Economics, 36(3), 441-455.

Vila, J. L., \& Zariphopoulou, T. (1997). Optimal consumption and portfolio choice with borrowing constraints. journal of economic theory, 77(2), 402-431.

Work, F. S. (2018). Grid Trading Strategy. https://www.valutrades.com/en/blog/how-to-tradecurrency-using-a-grid-strategy 